\title{
EVALUATING THE PERFORMANCE OF TERRESTRIAL LASER SCANNING FOR LANDSLIDE MONITORING
}

\author{
Mat Zam P.M, Fuad N.A, Yusoff A.R, and Majid Z
}

Geospatial Imaging and Information Research Group, Faculty of Built Environment and Surveying, Universiti Teknologi Malaysia - (en.pojie, nursyahiraaf, ahmadrazali89)@gmail.com, (zulkeplimajid)@utm.my

KEY WORDS: landslide monitoring, terrestrial laser scanning, scanning resolutions, geodetic method, deviation maps

\begin{abstract}
:
Nowadays, Terrestrial Laser Scanning (TLS) technology is gaining popularity in monitoring and predicting the movement of landslide due to the capability of high-speed data capture without requiring a direct contact with the monitored surface. It offers very high density of point cloud data in high resolution and also can be an effective tool in detecting the surface movement of the landslide area. The aim of this research is to determine the optimal level of scanning resolution for landslide monitoring using TLS. The Topcon Geodetic Laser Scanner (GLS) 2000 was used in this research to obtain the three dimensional (3D) point cloud data of the landslide area. Four types of resolution were used during scanning operation which were consist of very high, high, medium and low resolutions. After done with the data collection, the point clouds datasets were undergone the process of registration and filtering using ScanMaster software. After that, the registered point clouds datasets were analyzed using CloudCompare software. Based on the results obtained, the accuracy of TLS point cloud data between picking point manually and computed automatically by ScanMaster software shows the maximum Root Mean Square (RMS) value of coordinate differences were $0.013 \mathrm{~m}$ in very high resolution, $0.017 \mathrm{~m}$ in high resolution, $0.031 \mathrm{~m}$ in medium resolution and $0.052 \mathrm{~m}$ in $l o w$ resolution respectively. Meanwhile, the accuracy of TLS point cloud data between picking point manually and total station data using intersection method shows the maximum RMS values of coordinate differences were $0.013 \mathrm{~m}$ in very high resolution, $0.018 \mathrm{~m}$ in high resolution, $0.033 \mathrm{~m}$ in medium resolution and $0.054 \mathrm{~m}$ in low resolution respectively. Hence, it can be concluded that the high or very high resolution is needed for landslide monitoring using Topcon GLS-2000 which can provide more accurate data in slope result, while the low and medium resolutions is not suitable for landslide monitoring due to the accuracy of TLS point cloud data that will decreased when the resolution value is increased.
\end{abstract}

\section{INTRODUCTION}

Landslide is considered as one of the worst natural phenomenon that threat human life and property all over the world, including Malaysia. Some landslide moves slowly and cause damage steadily, and some landslide move so rapidly that can destroy property and take lives very suddenly and unexpectedly. In order to prevent landslide, an effective technique is required to maintain and monitor the slope area which is exposed to the risk of landslide. Nowadays, there are many method and approaches has been developed in different way and view to prevent and predict the landslide tragedy. The rapid growth in science and technology has introduced a new methodology and instrumentation in field of geomatics. One of the modern technology in surveying tools is Terrestrial Laser Scanning (TLS). The application of laser is adopted in the field of measurement and data capture in TLS has made it a different and unique technique compare to the other available methods.

Over the recent years, the interest in exploiting the TLS data for deformation monitoring has been increased. It has been reported by various researchers such as Lingua et al. (2008), Miller et al. (2008), Sui et al. (2008), Sui et al. (2009), Kasperski et al. (2010), Bertacchini et al. (2012) and Jing (2012) that the TLS is suitable for slope deformation and instability monitoring with the ability of providing dense three-dimensional (3D) point clouds in a short observing time without direct contact with the land slope surface. Furthermore, the accuracy of this technology is also acceptable for slope deformation monitoring with millimetre (mm) and centimetre (cm) level accuracy (Miller et al., 2008; Abellan et al., 2009; Barbarella et al., 2013). However, one of the aspects that require attention in the data acquisition is the optimum scan resolution (Miller et al., 2008; Monserrat and Crosetto, 2008). Therefore, a high-quality project planning is required to ensure the balance between the time used for data collection and the quality of the captured data. In addition, the resolution of the Digital Terrain Model (DTM) that can be generated for deformation analysis is directly influenced by the point cloud density. Hence, the aim of this research is to investigate the effect of different scanning resolution on landslide monitoring using TLS. Four types of scanning resolution are applied in this research in order to examine the suitability of optimum level for scanning resolution in land slope deformation detection.

Landslide can be triggered by natural environmental changes such as heavy rains, floods, vibrations from the earthquake and volcanic activities and either occurs as a result of human activities that disturb the slope stability. In order to prevent landslide, an effective technique is required to maintain and 
monitor the slope area and then can reduce the impact of this disaster. Over the years, slope monitoring activities are largely carried out by conventional survey instruments such as levelling, Total Station and Global Positioning System (GPS). However, these techniques are time-consuming, hazardous, costly process and sometimes difficult to be carried out because of the slopes surface is extent vertically and also difficult to be accessed due to the hazardous area (Wan Aziz et al., 2012). Hence, the laser scanning technology has become an ideal tool for landslide monitoring due to this equipment can serve without contact with the hazardous area and make it a helpful observation in landslide assessment.

Terrestrial Laser Scanning (TLS) enable the accurate and fast of data collection for millions of 3D point clouds data. Driven by more accurate and efficient sensor technology, laser scanning is nowadays accepted as a reliable method for accurate data collection of $3 \mathrm{D}$ point cloud data. In addition, the investigation of TLS performances has become a prominent topic of research in the last decades, so that a wide range of contributions about performance analysis of devices (Hanke et al., 2006), external parameter influences (Soudarissanane et al., 2011) and also accuracy of scanning resolution (Rosmadi et al., 2011) are available. Referring to previous research by Wan Aziz et al. (2012), Chen et al. (2010), Ching (2011), Yong (2012) and Mohd Rosydi (2013) there are numerous equipment of TLS have been used in landslide monitoring such as Leica ScanStation2, Topcon GLS-1500, Riegl LMS-Z620 and Faro Photon 120/20. However, until now there are no studies has been done related to landslide monitoring using Topcon GLS-2000 laser scanner. The recent Topcon GLS-2000 laser scanner offers a significant improvement in terms of scanning rate and point spacing resolution. With the capability of acquiring about 120,000-point cloud data per second and point spacing resolution which are consist of $3.1 \mathrm{~mm}, 6.2 \mathrm{~mm}, 12.5 \mathrm{~mm}$, $25 \mathrm{~mm}$ and $50 \mathrm{~mm}$, it can be an effective tool in detecting the surface movement of the landslide area. Therefore, utilization of Topcon GLS-2000 at different scanning resolution in landslide monitoring were experimented and evaluated in this research.

Wan Aziz et al. (2012) related on research about the slope gradient analysis at different scanning resolution using TLS, it was found that the scanning resolution affected slope map result. Hence, the scanning resolution must be set during the data scanning according to the application needs. The density of 3D point cloud data also depends on the scanning resolution of point clouds that used in TLS. However, high resolution required a long observation time for collecting the $3 \mathrm{D}$ point clouds data compared to low resolution. According to Miller et al. (2008) and Monserrat and Crosetto (2008), one of the aspects that require attention in the data acquisition is the optimum scan resolution. Therefore, a high-quality project planning is required to ensure the balance between the time used for data collection and the quality of the captured data. In addition, the resolution of the Digital Terrain Model (DTM) that can be generated for deformation analysis is directly influenced by the point cloud density. Based on the above review, the aim of this research is to investigate the optimum scan resolution for landslide monitoring using
Topcon GLS-2000 laser scanner. Four types of scanning resolution were applied in this research in order to examine the suitability of different types of scan resolution in slope deformation detection. Four types of scanning resolution were consisting of very high $(3.1 \mathrm{~mm}$ at $10 \mathrm{~m}$ distance), high (6.3mm at $10 \mathrm{~m}$ distance), medium $(12.5 \mathrm{~mm}$ at $10 \mathrm{~m}$ distance) and low $(25 \mathrm{~mm}$ at $10 \mathrm{~m}$ distance) resolutions. The different scanning resolutions were set during the scanning operation and this process were measured at two epoch observations. In general, the changes of slope geometry have been measured from several series of observation. The method of "point cloud to point cloud" comparison was used to calculate the deformation.

\section{LITERATURE REVIEW}

Landslide is a serious geologic hazard that happens in almost all states globally which lead to injury, death and also property and infrastructures damage (PWD, 2009). Based on the few types of landslide movement, the landslide which moves slowly will lead to steady damage while for the rapid landslide can be very sudden and unexpectedly destroy property and lives. The USGS described ten major landslide's type which not only can happen at hillside areas with steeps slope, but it also can happen at flat terrain which highly related with the material layer of the soil as shown in Figure 1.

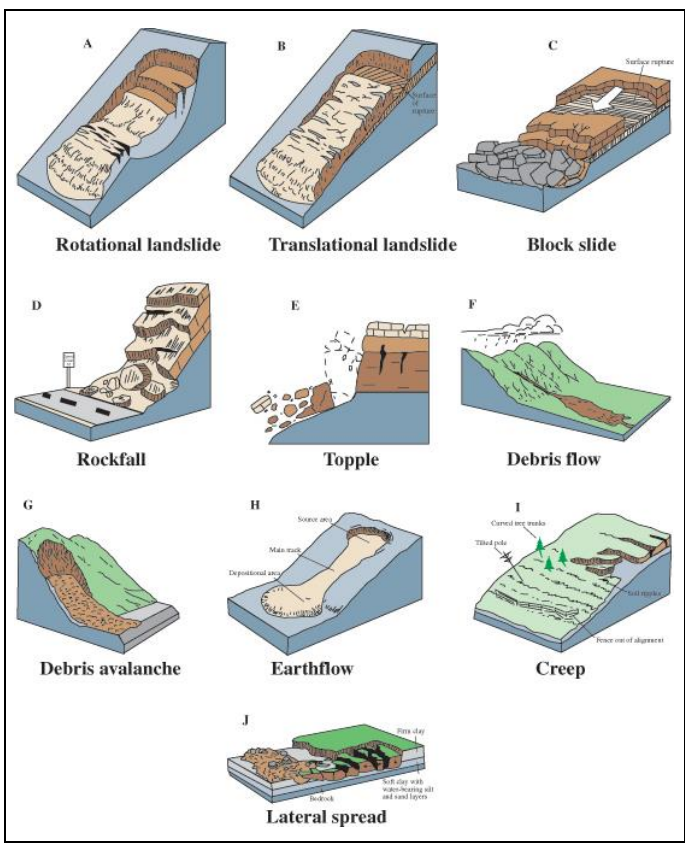

Figure 1. The Major Types of Landslide Movement (Source: Highland, 2004)

In Malaysia, PWD (2009) reported that most of the landslide events are shallow and small scale caused by surface infiltration or erosion during high intensity of rainfall especially in monsoon seasons (May to July in the northeastern region and October to December in the Southwestern region). This triggering factor increases the water content of the slope and reducing the materials strength. Such landslides 
are also related with poorly-maintained slope surface covers and drainage provisions. The raised water table after a rainstorm would saturate the soil rendering it weak and therefore the slope failure would naturally occur due to gravitational. Instead of natural forces, human activities such as deforestation due to uncontrolled development of hill slope areas are the main cause of landslides in Malaysia. In recent years, development at hillside area being highly demanded which offers the benefit of green surroundings, peaceful living conditions, and attractive views. Hence, the development of hillside area has growth up rapidly over the country. Excavation in the hilly areas has resulted to instability of the slope and causing numerous landslides tragedy.

Laser scanning is an advanced data acquisition method with rapid development since the mid-1990s. There are 2 types of laser scanning instrument which is airborne laser scanner and terrestrial laser scanner. Airborne laser scanners are located inside airplane and do the measurement from air to ground similar to aerial photogrammetric technique. On the other hand, terrestrial laser scanner is used at the ground in order to get the measurement (Anne, 2009).

According to Bohler and Marbs (2002), laser scanner measures the distances from sensor to the object using laser light in a systematic pattern. The data which called 'point cloud' can be turned into meshed 3D surface or models that useful for many applications such as surveying, reverse engineering, forensics, film and heritage restoration. The choice of laser scanner depends on applications. Some scanners are suitable for close range applications (up to some meters) with a high precision, indoor use with medium ranges (up to $100 \mathrm{~m}$ ), and some other scanners are better for outdoor use with long ranges (up to some 100m).

Laser scanner is categorised by which the range to an object is measured. This can be divide into several categorise depends on the principle of the laser scanning measurement system that use either time of flight, phase measurement or triangulation. Table 1 below shows the categories of laser scanner principle and from this table we can see the difference between range and accuracy of the measurement.

\begin{tabular}{|l|c|c|}
\hline Measurement Technology & Range (m) & $\begin{array}{c}\text { Accuracy } \\
(\mathrm{mm})\end{array}$ \\
\hline \multirow{2}{*}{ Time of flight } & $<100$ & $<10$ \\
\cline { 2 - 3 } & $<1000$ & $<20$ \\
\hline Phase Measurement & $<100$ & $<10$ \\
\hline Triangulation & $<5$ & $<1$ \\
\hline
\end{tabular}

Table 1. Categories of laser scanner principle

The basic principle of distance measurement is based on three main methods namely phase difference, time of flight (TOF) and triangulation. TOF method measures time taken for a laser pulse to travel to target and back. The distance is then computed based on the simple physic equation. The TOF posed its advantage in longest range measurement. The range can be achieved up to one kilometer which also depends on the type of the capability of the instrument. TOF however is less in accuracy compared with phase and triangulation method but still quite acceptable in which provides reliable output. Meanwhile, phase difference method measures the change in emitted phase and received phase of multiple sinusoidal laser pulses to determine distance. The phase measurement offers higher accuracy of distance measurement but the range is only over tens of meters. Another type of distance measurement is based on laser triangulation. This type of measurement is used for a very short distance measurement, which operates over range of a few meters only. It provides high degree of the accuracy, within fraction of millimeters (Syahmi, 2013).

Due to the capability of TOF to measure wide area from large range instrument setup, this type of measurement is being used widely in environmental survey as well as landslide investigation. Whereas, the phase differences method has a widespread used in close-range measurement to produce 3D object modelling (Lerma et al., 2009). Triangulation type is widely used in metrology, industrial application, reverse engineering, in body scanning, medical research and archaeology. The literature of both phase differences and triangulation methods is however out of the scope, where the emphasis is exclusively on the larger range of measurement that fit the landslide research and commonly used in natural environment application.

Time of flight (TOF) measurement is also known as time pulse method or pulse echo method. The measurement is based on very accurate time measurement of a short pulse to travel from the sensor to the object, reflected and return to the sensor (Figure 2). Based on the principle of velocity in physics, the distance of the target or point can be computed since the velocity of the laser light is accurately known.

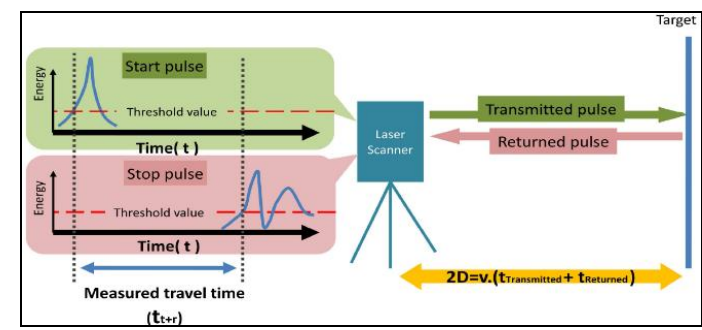

Figure 2. The basic principles of distance measurement of TLS based on TOF method (Source: Modified from Petrie and Toth, 2008)

Based on the figure above, the distance of the object can be computed by;

$$
D=v \cdot t / 2
$$

where,

$\mathrm{D}=$ slope distance

$\mathrm{v}=$ speed of electromagnetic radiation

$\mathrm{t}=$ measured time of travelling or time of flight

Since the distance can be computed accurately, the threedimensional coordinate of the point can be determined. In scanning mechanism, utilizing the rotating mirror and motor drive in the instrument allows laser pulse transmitted in 
vertical and horizontal direction respectively. By assuming the sensor's position is $(0,0,0)$, the three dimensional of the point position thus can be computed using the slope distance measured in previous equation and also angular measured in vertical and horizontal of the point. Therefore, the basic trigonometric equation can be applied based on Figure 3 .

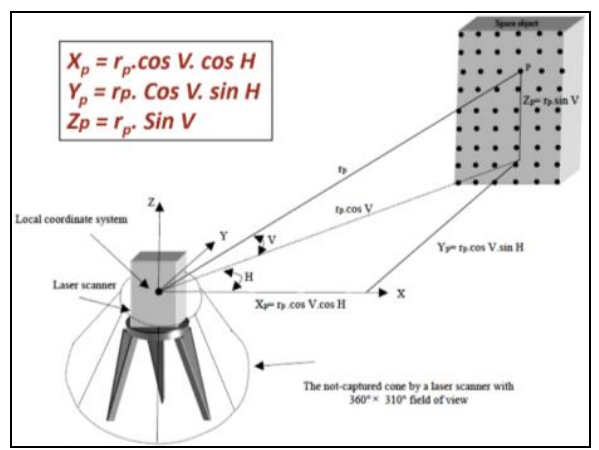

Figure 3. How Terrestrial Laser Scanner Works (source: Zulkepli, 2015)

This principle is used to improve precision in distance measurement. This technique is based on phase comparison method which is Electronic Distance Measurement transmits a coaxial intensity modulated optical measuring beam that is reflected off a target. It can be defined as the phase difference between the transmitted light and the reflected light represents a distance. However, only the phase offset obtains from the phase comparison (Roshan and Christoph, 2010). This is similar to time of flight detection except that the phase of the reflected laser light further refines the distance detection. In the phase difference method, the phase shift $(\Delta \Phi)$ between the sent and the received signal with a certain wavelength $(\lambda)$ is determined (Figure 4). The computation of the required distance is depending on the phase shift (Abdelhafiz, 2000).

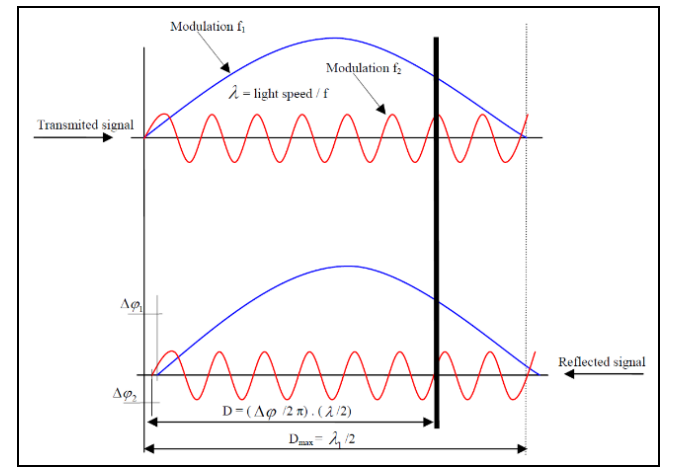

Figure 4. Phase shift scanning method (source: Abdelhafiz, 2000)

The idea of triangulation is having two laser beams instead than one. It is because the laser beam produced by either two separate lasers. Then, the angle of intersection between two laser beams is measured and absolutely we can calculate the distance to target object. The principle of laser triangulation system is a laser projector shines a thin sheet of light onto the object and then, CCD sensor detects on each scan line which is the peak of reflected laser light. CCD sensor is located at a known distance from the laser's source. Finally, 3D point positions or called as point cloud that contains 3D information, $\mathrm{X}, \mathrm{Y}, \mathrm{Z}$ value are computed using the intersection of the line through the pixel with the known plane of laser light. Figure 5 shows the principle of the laser triangulation system according to Bernardini and Rushmeier (2002).

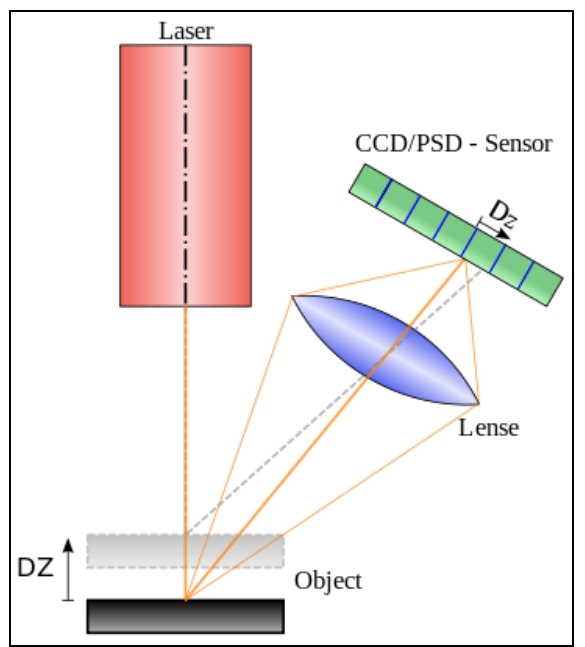

Figure 5. Principle of triangulation (source: Abdelhafiz, 2000)

According to Boehler and Marbs (2003), the term of resolution is used in different contexts when discussing the performance of laser scanner. From a user perspective, the resolution describes the capability to detect either small objects or object features in the point cloud data. Technically, two different specifications of laser scanner contribute to this capability, the size of the laser spot itself on the object and the smallest possible increment of the angle between two successive points. Most laser scanner allow the users to perform manual settings of the increment.

Before the scan resolution is decided, the behaviour of the scanner shall be first understood, that is related with the scene range, sample spacing and number of points recorded. Scene range is a representative distance from the scanner location to the scene to be scanned. Sample spacing displays distance between two adjacent points in a specific range. While, the number of point displays the total number of point clouds in horizontal and vertical directions, based on the selected range and sample spacing. The dependencies between the resolution setting elements are; changing range changes number of points based on sample spacing, changing sample spacing changes number of points based on range, changing number of points changes sample spacing based on range. In other words, by increasing the range, the number of points emitted will be increased in order to achieve the desired sample spacing. The number of points will be automatically determined by the scanner while the range and the sample spacing will be based on the user settings (Syahmi, 2013).

The important thing of the scanning parameter which can affect the resolution before starting the scanning is point spacing also known as angular resolution. The function of the stepping angle (increments of pulses for each set) for the laser 
and the range to the target can increase or decrease the point spacing by adjusting the stepping angle also known as angular step in the scanning software. This will result in reducing or increasing the resolution respectively and the corresponding density of point cloud which were collected by the scanner (Figure 6). When the distance is increase the point spacing of point cloud will also increase. Therefore, the angular step should be reduced in order to obtain a high-resolution in data scanning at long distance measurement. In addition, the suitability of the angular step for both vertical and horizontal can be adjusted depending on the project resolution requirements and the amount of data to be collected and analysed. Very high density of point clouds requires more space for data storage and requires more power for processing on computers, and it also take a longer time for data scanning. This will affect the time taken for data collection in field and also how long it will take for data processing, so it should be considered in the project design (Carr et al., 2013).

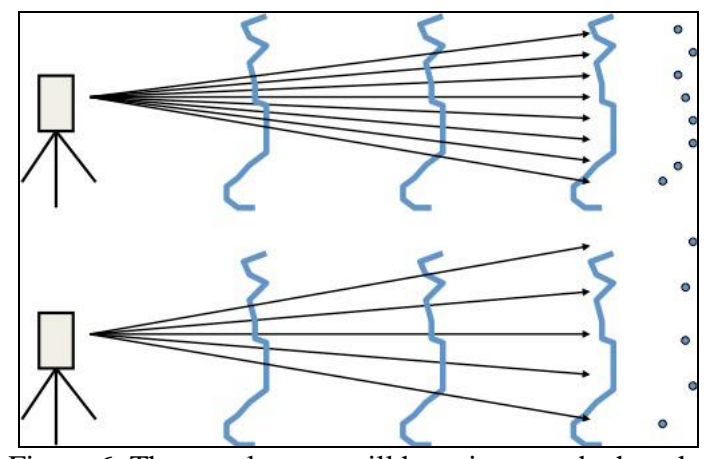

Figure 6. The angular step will have increased when the distance of the scanner is increased (source Carr et al., 2013)

The application of laser scanners in natural environment has been widely conducted as well as mass movement investigation-see Jaboyedoff et al. (2010) for the review of the current status. Since the laser scanner permits high density of observation, more realistic three-dimensional digital terrain model (3D DTM) can be produced. With reflectorless technology laser measurement, TLS is able to be conducted in limited accessibility area as well as the landslide area. Therefore, utilization of laser scanning technology in the landslide investigation could be experimented and evaluated further to prove its capabilities in landslide research.

Recently, Terrestrial laser scanner (TLS) have been used for data collection of 3D point cloud data in order to maintain and monitor the movement of landslide area. In addition, high resolution in TLS can provide a high density of point cloud data. Referencing to Monserrat et al. (2008) was proposed a new procedure in detecting the movement of the landslide area using TLS. In this research, laser scanner has already proven to provide accurate result in landslide deformation. Theoretically, TLS can provide a very high redundancy data which allows the detection of landslide movement in centimeter level. There are many previous techniques has been used to detect the movement of landslide area which are consist of levelling, total station, global positioning system, satellite image, close range photogrammetry and airborne laser scanner (Tsakiri et al. 2006). The accuracy of all these techniques offer a different slope result. Usually the landslide phenomenon occurs due to mass movement between $0.01 \mathrm{~mm}$ to $10 \mathrm{~mm}$ daily (Miyazaki et al. 2008). Thus, TLS is the best solution in detecting the landslide movement and it can also provide the data until to millimeter level (Sui et al. 2008).

This section discussed the issues in landslide investigation and monitoring, and the basic principles of TLS measurement which are practically conducted based on geomatics field. In addition, the scanning resolution of TLS was discussed in order to relate the scan resolution and the accuracy of the surface model for any other application. The next chapter will focus on describing the instruments and software which are used in order to achieve the objectives of this research.

\section{METHODOLOGY}

The methodology of this research consists of three major phases. These include fieldwork strategy, data acquisition and data processing. The detail description of the research workflow is described in the following sections.

\subsection{Field Survey Strategies}

The most important thing in this research is field survey strategies which aims to avoid revisit the site. Excellent survey planning will contribute to smooth data acquisition, hence, realize the efficiency of TLS performance. For that purpose, several approaches in carrying out TLS fieldwork has been identified and proposed as shown in Figure 7.

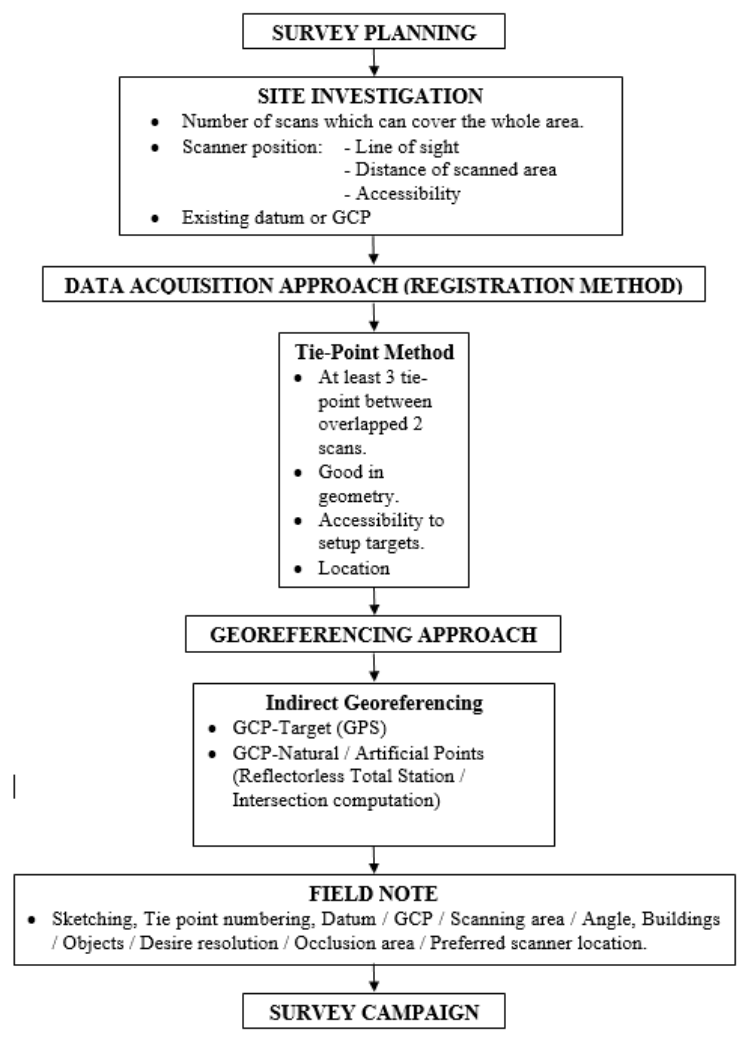

Figure 7. Field survey strategy workflow 


\subsection{Data Acquisition}

The data has been acquired in two time-series or epochs over the same area. The first observation has been carried out on 5 September 2017. Later, the observation was repeated for the second epoch on 7 November 2017. Both epochs were conducted in a similar manner whereby instrument setup (i.e. scanner position, scan resolution) were maintained and used during scanning operation in order to reduce errors from the instrument handling and inconsistencies of the point clouds distribution within both observations.

In this research, four different types of resolution were used during scanning operation and they are consist of very high resolution $(3.1 \mathrm{~mm}$ at $10 \mathrm{~m}$ distance $)$, high resolution $(6.3 \mathrm{~mm}$ at $10 \mathrm{~m}$ distance), medium resolution $(12.5 \mathrm{~mm}$ at $10 \mathrm{~m}$ distance) and low resolution (25mm at $10 \mathrm{~m}$ distance). Concurrently, the scan distances between slope surface and observation stations is $35 \mathrm{~m}$ approximately and the length between two observation stations is $30 \mathrm{~m}$ approximately. On the other hand, all the control points were well-distributed around the deformation area. These control points are used for registration purpose. Meanwhile, all the target sheets were located inside the area of interest and this area is considered unstable. Figure 8 shows the overall layout of the control points, target sheets and observation stations.

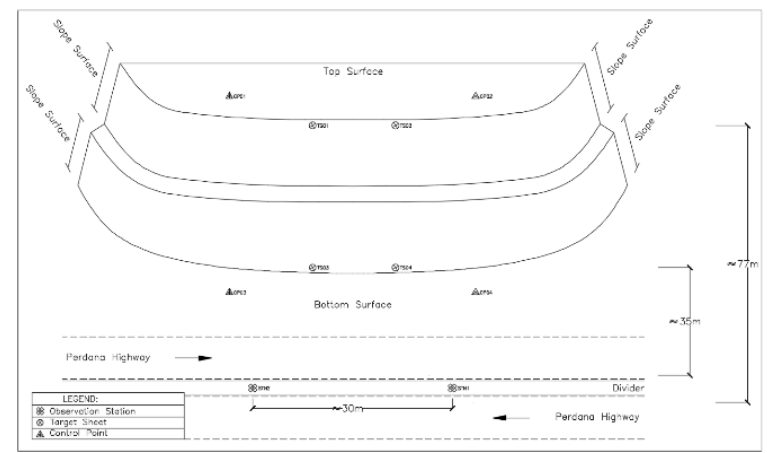

Figure 8. Overall layout of the control points and observation stations

The data acquisition conducted in this research consists of three major phases; (i) GPS observation (ii) intersection method and (iii) scanning process. The details of the task are described in the following sections.

\subsection{GPS Observation}

In this research, two observation station were established on the middle of the divider road opposite the land slope. All the observation stations were established with PVC pipe and concrete (Figure 9). The observation stations have been observed before the scanning process by using Topcon Hiper V GNSS receiver (Figure 10). The type of observation used is Rapid Static control survey with post processed (correction) mode derived through Virtual Reference Station (VRS) from MyRTKnet infrastructure. Each observation stations have been observed for 30 minutes. This observation has been done in two time-series or epochs for the same observation stations.

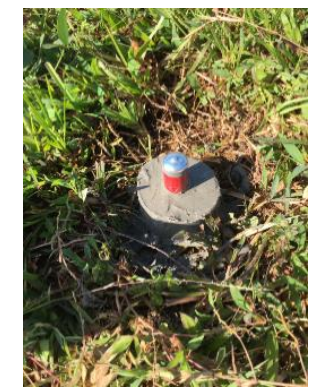

Figure 9. The example of observation stations established

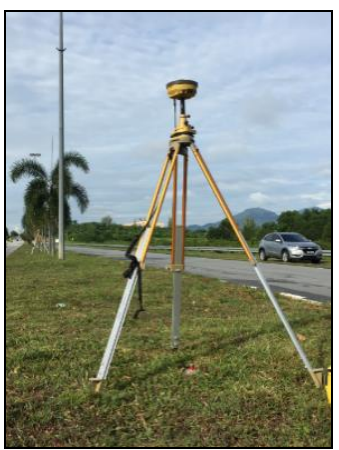

Figure 10. Topcon Hiper V GNSS receiver is used to obtain the coordinate value of observation stations

All the observation stations have been processed through Topcon Tools software version 8.2.3 (Figure 11) and the final coordinates are shown in Figure 4.6. All the coordinate value of observation stations is converted in RSO coordinate system. Table 2 shows the differences of observation stations coordinate value between both epochs.

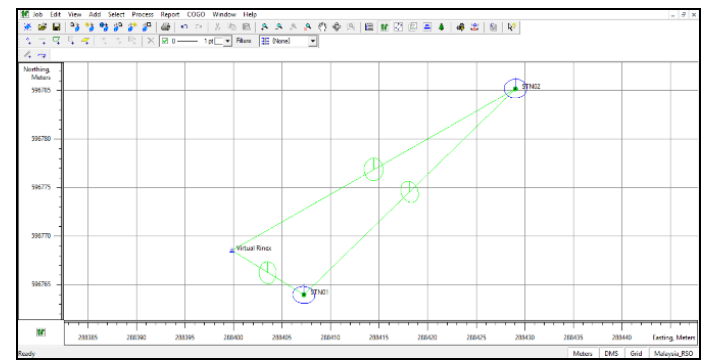

Figure 11. The example of post processed for using Topcon Tools software version 8.2.3

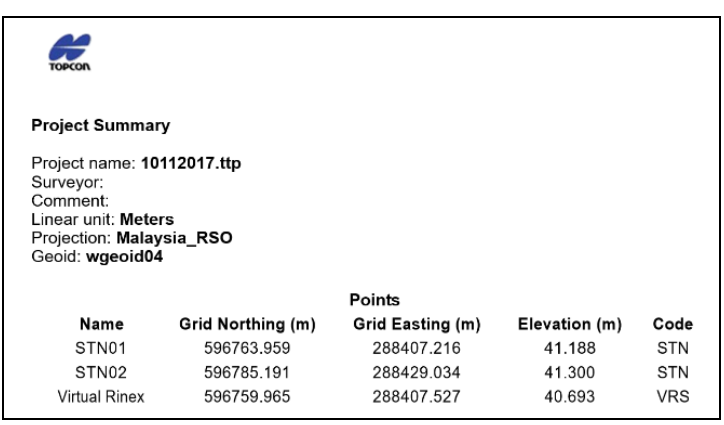

Figure 12. The example of final coordinates after post processing using Topcon Tools software 


\begin{tabular}{|c|c|c|c|c|c|c|c|c|c|}
\hline \multirow{2}{*}{$\begin{array}{l}\text { Observation } \\
\text { Stations }\end{array}$} & \multicolumn{3}{|c|}{ Epoch 1} & \multicolumn{3}{|c|}{ Epoch 2} & \multicolumn{3}{|c|}{ DIFFERENCES } \\
\hline & Easting (m) & Northing $(m)$ & Height (m) & Easting (m) & Northing (m) & Height (m) & $\Delta E(m)$ & $\Delta \mathrm{N}(\mathrm{m})$ & $\Delta \mathrm{H}(\mathrm{m})$ \\
\hline STN01 & 288407.216 & 596763.959 & 41.188 & 288407.219 & 596763.961 & 41.193 & -0.003 & -0.002 & -0.005 \\
\hline STN02 & 288429.034 & 596785.191 & 41.300 & 288429.032 & |596785.187 & 41.294 & 0.002 & 0.004 & 0.006 \\
\hline & & & & & & Max & 0.002 & 0.004 & 0.006 \\
\hline & & & & & & Min & -0.003 & -0.002 & -0.005 \\
\hline & & & & & & Average & 0.000 & 0.001 & 0.001 \\
\hline & & & & & & RMS & 0.002 & 0.003 & 0.005 \\
\hline
\end{tabular}

Table 2. Coordinates value of observation stations for both epochs and its differences

\subsection{Intersection Method}

In this research, all the control points were established outside of the interest area with PVC pipe and concrete (Figure 13). Furthermore, all the control points were well-distributed around the deformation area. These control points are used for registration purpose. On the other hand, all the target sheet is used for checks point on the slope area surface because all points were located inside the interest area and this area is considered unstable. Figure 14 shows the overview of the distribution of the control points and the target sheets on the land slope.

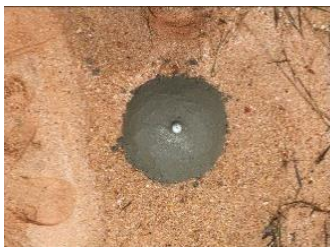

Figure 13. The example of control points established

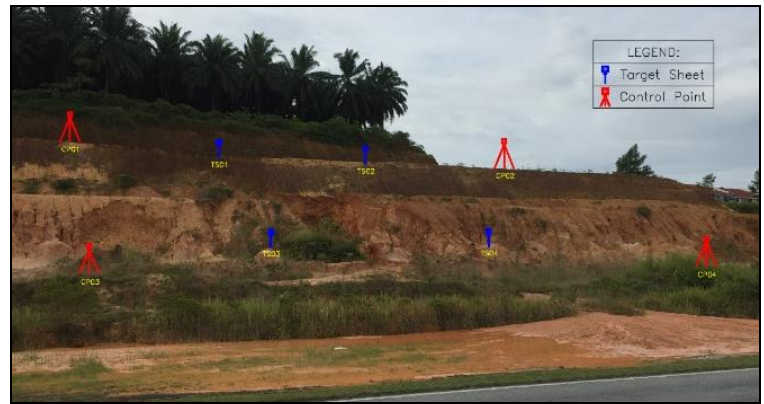

Figure 14. The distribution of the control points and target sheets on the land slope

Once the coordinate values of both observation stations were obtained through GPS observation, all the control points and target sheets were observed from the two known observation stations using the total station Topcon GTS-226 (Figure 15). All the control points and target sheets were observed before the scanning process in order to obtain 3D coordinates (easting, northing and height).

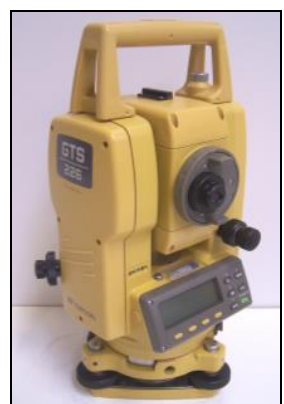

Figure 15. Total Station Topcon GTS-226

The surveying intersection concept was applied in the measurements and the $3 \mathrm{D}$ coordinates of the measurement target scans were calculated using the Allan formula. This process validates the quality of the scanned data. Figure 16 below shows the surveying intersection concept.

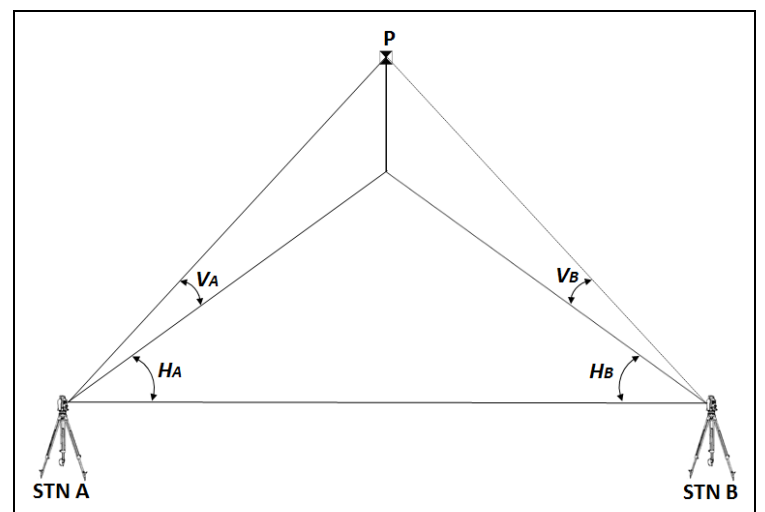

Figure 16. Surveying intersection concept - where P, HA and $\mathrm{HB}$, and VA and VB are the measurement target, horizontal angles and vertical angles, respectively (source: Zulkarnaini, 2016)

The computational for Allan's Formula are:

$$
\begin{array}{ll}
\begin{array}{l}
\text { Coordinate of } \mathrm{P} \\
E_{p}=E_{1}+l_{1} t_{1}
\end{array} & \begin{array}{l}
\text { Coordinate of } \mathrm{P} \\
E_{p}=E_{2}+l_{2} t_{2}
\end{array} \\
N_{p}=N_{1}+m_{1} t_{1} & N_{p}=N_{2}+m_{2} t_{2} \\
Z_{p}=Z_{1}+n_{1} t_{1} & Z_{p}=Z_{2}+n_{2} t_{2} \\
\text { Where: } & \\
l_{1}=\operatorname{Cos} V_{A} \times \operatorname{Sin} H_{A} & l_{2}=\operatorname{Cos} V_{B} \times \operatorname{Sin} H_{B} \\
m_{1}=\operatorname{Cos} V_{A} x \operatorname{Cos} H_{A} & m_{2}=\operatorname{Cos} V_{B} \times \operatorname{Cos} H_{B} \\
n_{1}=\operatorname{Sin} V_{A} & n_{2}=\operatorname{Sin} V_{B}
\end{array}
$$




$$
\begin{aligned}
& t_{1}=\frac{p+q \times \operatorname{Cos} C}{\operatorname{Sin}^{2} C} \\
& t_{2}=q+t_{1} \times \operatorname{Cos} C \\
& p=l_{1}\left(E_{B}-E_{A}\right)+m_{1}\left(N_{B}-N_{A}\right)+n_{1}\left(Z_{B}-Z_{A}\right) \\
& q=-l_{2}\left(E_{B}-E_{A}\right)-m_{2}\left(N_{B}-N_{A}\right)-n_{2}\left(Z_{B}-Z_{A}\right) \\
& \operatorname{Cos} C=l_{1} \times l_{2}+m_{1} \times m_{2}+n_{1} \times n_{2}
\end{aligned}
$$

This observation has been carried out in two epochs from the same observation stations. Figure 17 below shows the observation of total station using intersection method from two observation stations at research area. Both of these epochs of data collection have been processed using the Allan formula, which has been formulated in Microsoft Excel. The differences of intersection result between both epochs are shown in Table 3. Based on the results obtained, the marked point of target sheets shows more movement occurred. This is because the target sheets were located inside the slope area and considered unstable compared to control points were well distributed outside of the slope area.

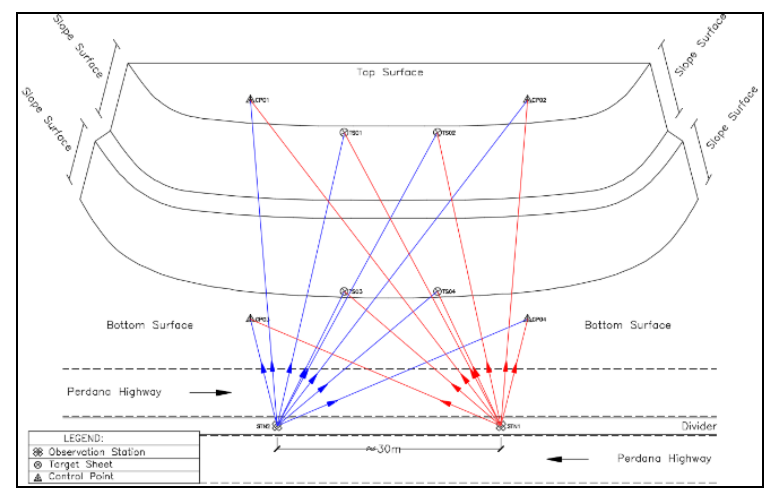

Figure 17. The observation of total station using intersection method from two observation stations at research area

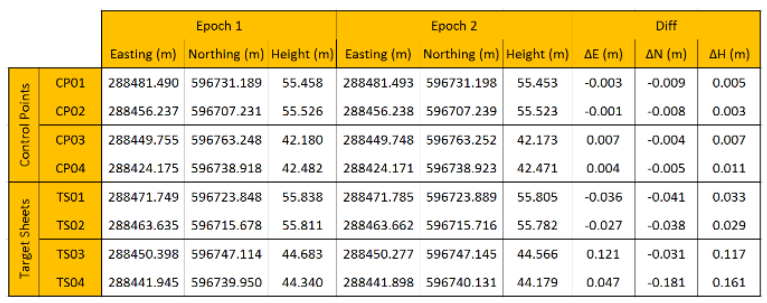

Table 3. The differences of intersection result between both epochs

\subsection{Scanning Process}

There are two scanning techniques that can be used to measure the slope surface which are consist of Tie-point method and Traverse method by using Topcon GLS-2000 (as in Figure 18). Tie-point method is the technique that stitching by at least three common points (tie-point) between overlapped two scans.

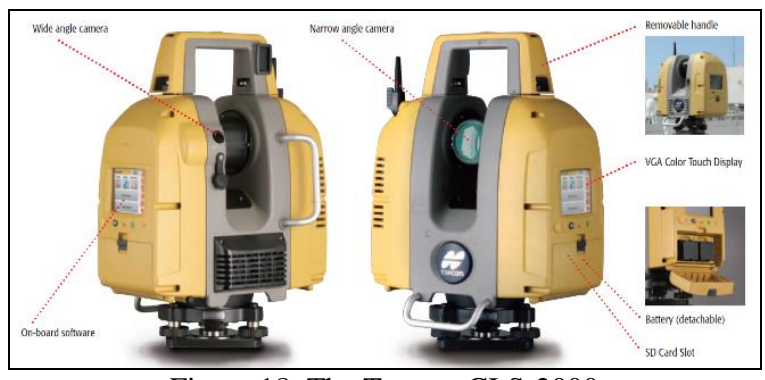

Figure 18. The Topcon GLS-2000

Then, if the tie-points have survey coordinates, stitched scans are calculated to survey coordinates. Meanwhile, traverse method (also known as Occupation / Backsight method) is one of the technique that can be conducted in a similar manner to general survey method such as traversing. The occupied station of laser scanner and backsight station must be setup on the known point which have been obtained from the traverse or GPS survey for direct approach. The survey coordinates of scanning data for this method are calculated directly relative to the known point. The procedure of scanning process is shown in Figure 19.

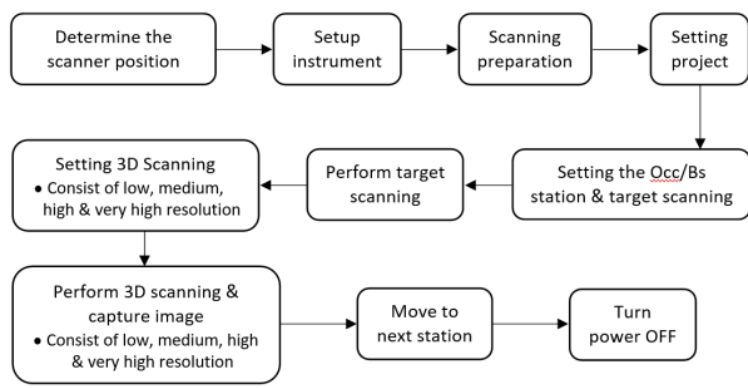

Figure 19. Procedure of scanning process

In this research, four different types of resolution were used during scanning operation and they are consist of very high resolution $(3.1 \mathrm{~mm}$ at $10 \mathrm{~m}$ distance), high resolution $(6.3 \mathrm{~mm}$ at $10 \mathrm{~m}$ distance), medium resolution $(12.5 \mathrm{~mm}$ at $10 \mathrm{~m}$ distance) and low resolution $(25 \mathrm{~mm}$ at $10 \mathrm{~m}$ distance). The data has been acquired in two epochs over the same area. Both epochs were conducted in a similar manner whereby instrument setup (i.e. scanner position, scan resolution) were maintained and used during scanning operation in order to reduce errors from the instrument handling and inconsistencies of the point clouds distribution within both observations. Generally, the slope area has been scanned in two different scanner locations. Figure 20 shows the scanner view of the slope taken from different scanner location which comprises of station 1 and 2 . 


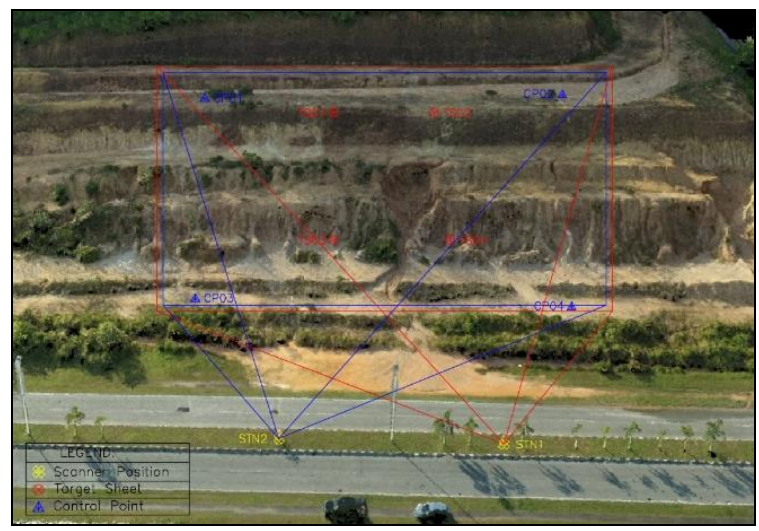

Figure 20. Scanner view of the slope taken from different scanner location which comprises of station 1 and 2

The target sheets of GLS-2000 manufactured by Topcon with special designed straw board holder were planted on the surface of the land slope when the data collection was carried out using Topcon GLS-2000 laser scanner. Besides that, the prism also used as target scan in this research which were placed on the control point. Figure 21 shows the type of targets scan used during the scanning process.

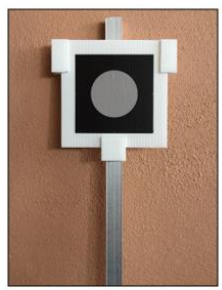

Target sheet

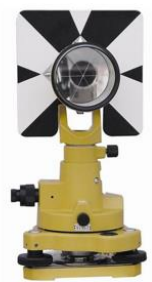

Prism
Figure 21. Types of target scan used during the scanning process

There are three types of target sheet manufactured by Topcon such as large, medium and small size (Figure 22). The selection of target sheet is based on the scanning distance (Table 4). For this research, a medium target sheet was used as target scan because the distance between the scanner station and the object less than 100 meter.

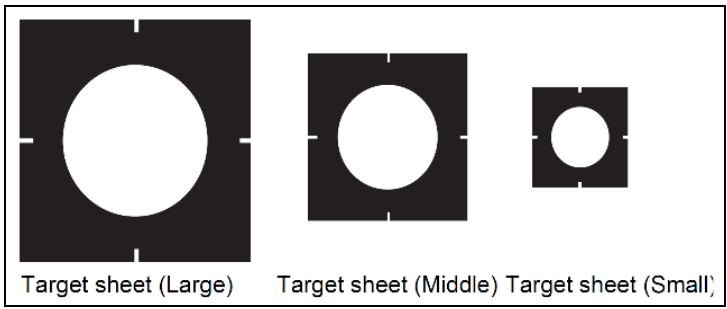

Figure 22. Three types of target sheet manufactured by Topcon

\begin{tabular}{|c|c|c|}
\hline Target & $\begin{array}{c}\text { Distance to the } \\
\text { scanning target }(\mathbf{m})\end{array}$ & Size $(\mathbf{m})$ \\
\hline Target sheet (Large) & $50-200$ & 0.12 \\
\hline Target sheet (Medium) & $10-100$ & 0.06 \\
\hline Target sheet (Small) & $2-50$ & 0.03 \\
\hline
\end{tabular}

Table 4. The selection of target sheet is based on the scanning distance

After done with all preparation to perform scanning, firstly, the setup and levelled the scanner very precisely to the observation station and then turn the power ON. The scanner was leave for a while and it takes three minutes after the power has been switched ON to initialize the instrument and warm up the laser. Then, create new project. All the scanned data are managed and stored into one project. After that, before start the scanning, create a new station and make sure to create a new station when moving to another station. Figure 23 shows the example of data configuration for scanner station which are stored in SD card.

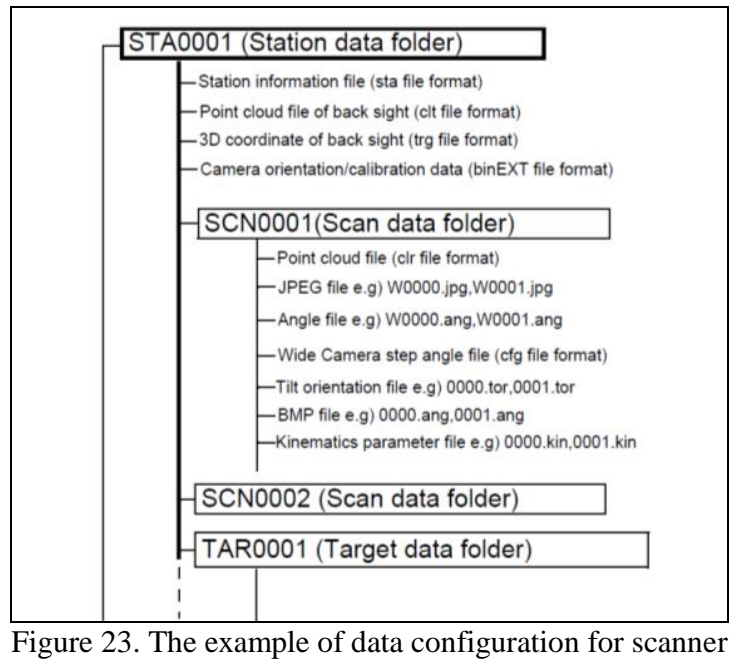

station are stored in SD card

There are two ways that should be done to perform scanning which are consist of target scanning and 3D scanning. The target scanning and 3D scanning process are briefly described in the following section.

\subsection{Target Scanning}

The target scanning has been performed to align the positional information of multiple 3D scanning data to perform post-processing of data. Thus, the target scan has been placed in a position that can be seen from the position of the next station. Only the prism or target sheet manufactured by Topcon can be used as the target scan during the scanning process and at least three set of common targets scanned data were required to align the positional information of data. In this research, four target sheets and four prisms were used as target scan which were distributed around the slope area. The scanning results of the target scan using Topcon GLS-2000 will be displayed as shown in Figure 24. The target scan data is added to the coordinate point list automatically. Similarly, same steps were performed until the completion of target scanning. 


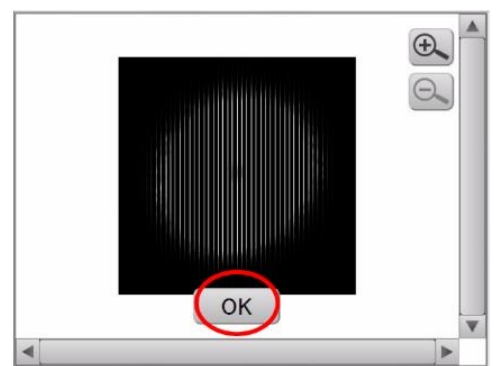

Figure 24. The display of scanning result for target scan

\subsection{D Scanning}

After setting up all the control points and the target sheets, the Topcon GLS-2000 laser scanner was used to scan the slope surface from two scan stations. There are two types of 3D scanning which are consist of range setting scanning and whole circumference scanning. In this research, the range setting scanning technique was used to $3 \mathrm{D}$ scanning. This technique can avoid the longer time consumed by scanning at the areas that are not interested. The boundary of the scan area is directly defined in the interface of the instrument by specifying the left top and right bottom of scanning area (Figure 25). In this research, the entire scanning process has been successfully completed from two scanner stations in different scanner standpoint (Figure 26) and each scanner station have four scanning data in difference resolution which were consist of very high resolution $(3.1 \mathrm{~mm}$ at $10 \mathrm{~m}$ distance), high resolution $(6.3 \mathrm{~mm}$ at $10 \mathrm{~m}$ distance), medium resolution $(12.5 \mathrm{~mm}$ at $10 \mathrm{~m}$ distance) and low resolution $(25 \mathrm{~mm}$ at $10 \mathrm{~m}$ distance). The whole time consuming for 3D scanning process approximately at three to four hours in this survey campaign.

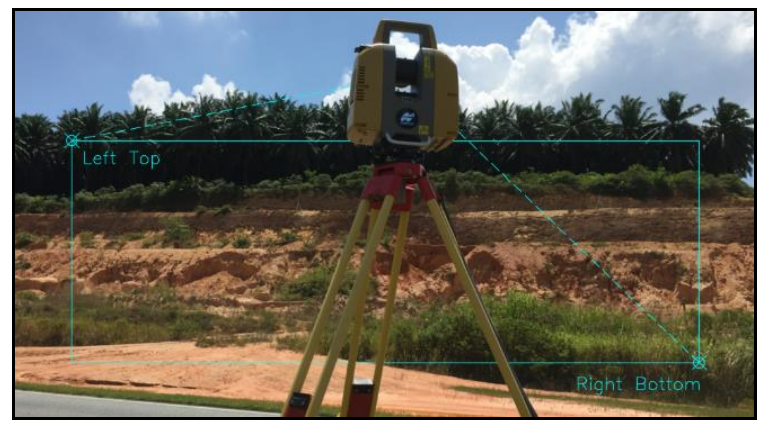

Figure 25. Determine scanning area
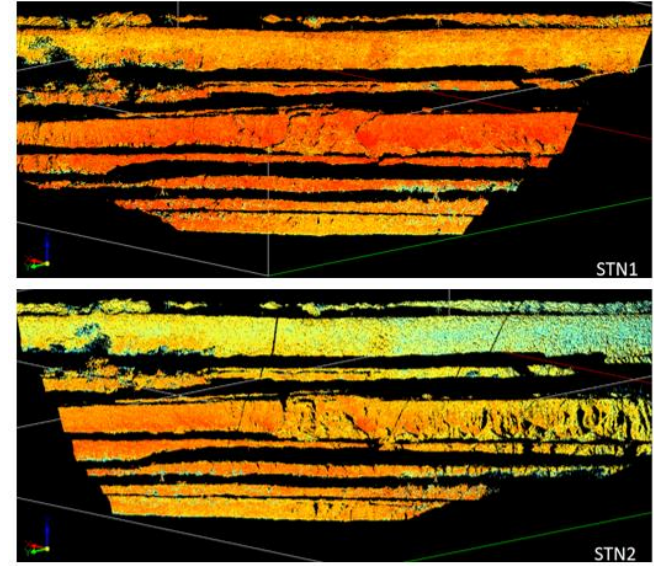

Figure 26. The difference scanner standpoint from two scanner stations

\section{8}

\section{Data Processing}

This section briefly describes data processing stages that has been used in this research. Generally, the workflow of the data processing stage is almost similar with other post-process TLS system. It starts with registration of the multiple scanned, then followed by georeferencing process. Afterwards, the data undergoes several processes to produce 3D DTM as a final output that was analysed later. In this research, ScanMaster software was used to perform the data registration, georeferencing and data filtering. After that, the registered point clouds datasets from two epochs were analyzed using CloudCompare software in order to calculate the deformation of land slope by applying the "cloud to cloud" computation technique. The general procedures of data processing for both software are briefly described in following section.

\subsubsection{Data Processing Using ScanMaster Software}

This section describes the data processing stages which conducted using ScanMaster Software. There are few steps involved which are consist of data checking, data registration, georeferencing, data colouring, data filtering and until it was done with editing. Figure 27 below shows the processing steps of $3 \mathrm{D}$ point cloud data from the beginning project until it was done with editing.

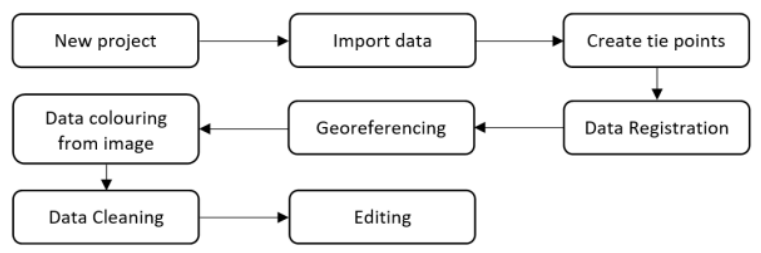

Figure 27. Data processing steps using ScanMaster software

\subsubsection{Registration of Multiple Scans}

When the data was captured from two different scan positions such as station 1 and station 2, the data is not exactly aligned. Figure 28 below shows the example of imported data which were obtained in this research were not oriented and aligned 
after the scan data was imported. This is because the data collection from the scan position are recorded in the local coordinate system of the scanner and the scanner cannot determine that it was moved to a new position, so it does not align the data. Thus, registration is the process of aligning the data using tie points from multiple scan positions. This process is important to align the data taken from different scan positions into the same global coordinate system. To permit the registration process, each scan shall consist of sufficient number of tie-point that is minimum of three tiepoints. Figure 29, shows the data after registration were oriented and aligned from from multiple scan positions. At this stage, all scans are combined and aligned as closely as possible to produce one coherent scan.

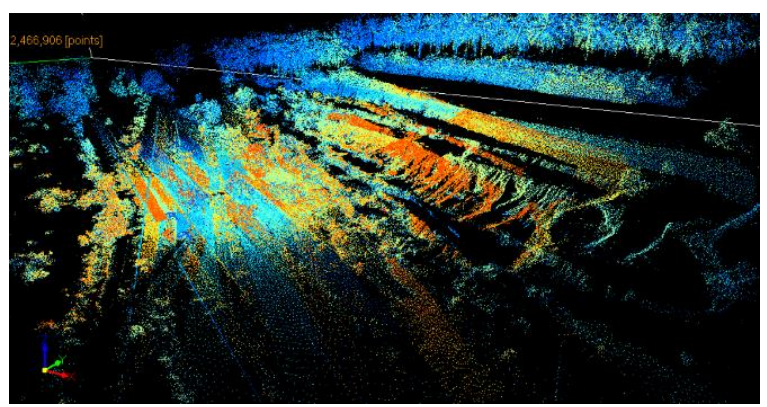

Figure 28. Data before registration was not aligned after the scan data was imported

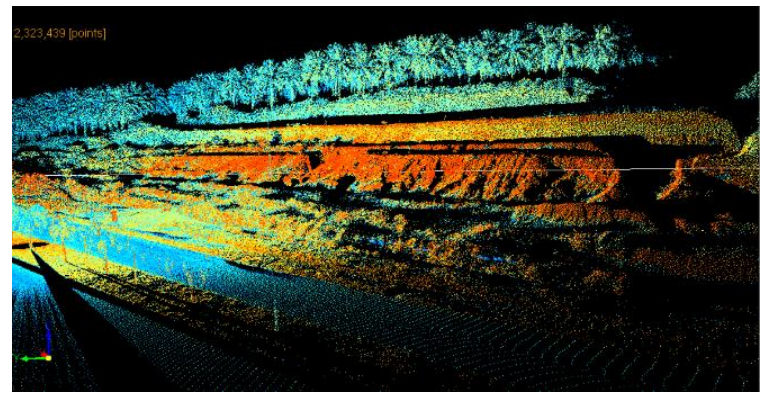

Figure 29. Data after registration was oriented and aligned from multiple scan positions

\subsubsection{Georeferencing}

Georeferencing enables to reorient the entire dataset to the corresponding coordinates of the tie point constraints measured using a GPS or total station with the coordinates from laser scanner. In this research, the registered point clouds were prepared for georeferencing process where the scanner coordinate systems were transformed into Rectified Skew Orthomorfic (RSO) coordinate system. In this process, all the coordinates value of control points and target sheets that obtained from total station observation and have value in RSO coordinate system were used and created. Figure 30 shows all the new points that were created appears in the Points folder in the Project Explorer. After finished, the created points were associated to the corresponding tie point constraints. Figure 31 shows the corresponding points that were associated from the Georeferenced drop-down menu.

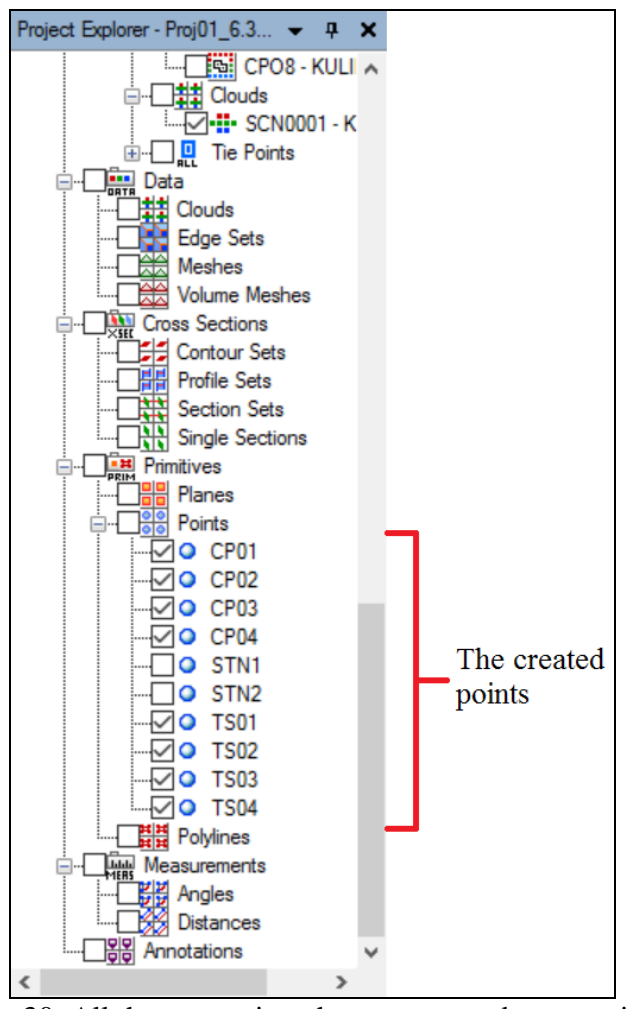

Figure 30. All the new points that was created appears in the Points folder

\begin{tabular}{|c|c|}
\hline \multicolumn{2}{|l|}{ Object Properties } \\
\hline \multicolumn{2}{|l|}{ 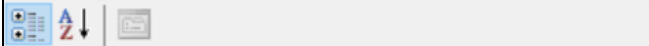 } \\
\hline \multicolumn{2}{|l|}{$\Xi$ Display } \\
\hline Layer & $\mathbf{0}$ \\
\hline \multicolumn{2}{|l|}{$\boxminus$ General } \\
\hline \multicolumn{2}{|l|}{ Description } \\
\hline Name & Tie Point Constraint 01 \\
\hline 田 Position & 288481.479: 596731.193: 55.456 \\
\hline \multicolumn{2}{|l|}{$\boxminus$ Georeference } \\
\hline Georeference & 2 \\
\hline \multicolumn{2}{|l|}{ Georeference Emor } \\
\hline$\boxminus$ Registration & СР01 \\
\hline Active & CP02 \\
\hline$\boxminus$ Tie Points & CPO4 \\
\hline Tie Point Count & CPO3 \\
\hline ( Tie Point Error - Maximum & TS01 \\
\hline 田 Tie Point Eror - Mean & TSO2 \\
\hline & TSO3 \\
\hline & STN1 \\
\hline & STN2 \\
\hline & \\
\hline
\end{tabular}

Figure 31. The corresponding points that were associated from the Georeferenced drop-down menu

\subsubsection{Data Coloring from Image}

For the next step is colouring the data from the images which is important to improve the better sense of what was captured with the laser scanner. There are seven modes of colour technique which are elevation, image, intensity (BW), intensity (RGB), intensity (RYGCB), layer and normal. The right colour was chosen to manage the point clouds data. 
Figure 32 shows the color matching from the image (digital photographs) that was completed in order to make the $3 \mathrm{D}$ point cloud model exactly the same with real object.

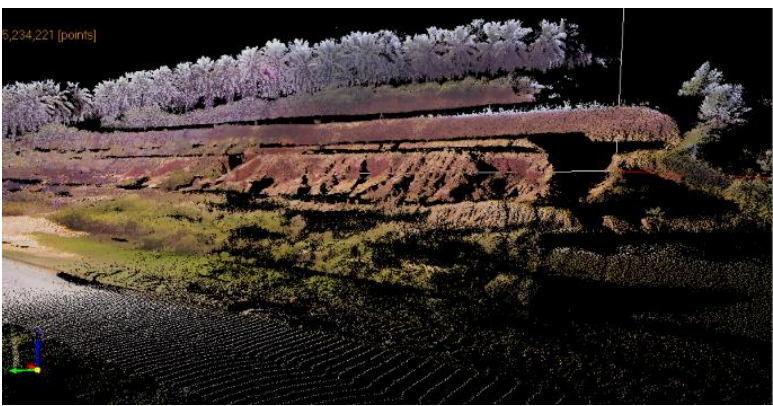

Figure 32. After finished the colour matching from the image

\subsubsection{Data Cleaning}

The final data processing using ScanMaster software is data cleaning. This process was used to clean georeferenced point clouds from any unrecognized or unused point clouds such as trees, vehicle, and other unwanted point clouds. The ScanMaster software provides several tools to help data cleaning process especially in obtaining ground surface. Figure 33 shows the unnecessary scanned data was selected using Polygon Selection icon on toolbar and then the unnecessary data was deleted using Delete Data icon on toolbar.

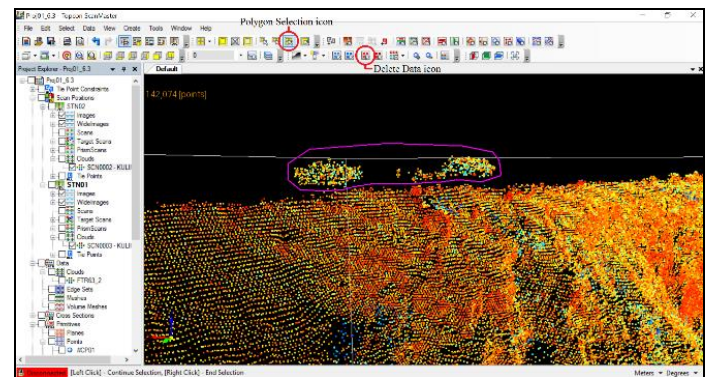

Figure 33. Unnecessary scanned data was selected using Polygon Selection icon and was deleted using Delete Data icon on toolbar

In addition, other methods that can be used in ScanMaster software to efficiently remove such irregular noises is Filtering Noise method. This function extracts and selects isolated point and irregular noise by referring point density of local regions. Figure 34 shows the Filter Noise on the shortcut menu. By adjusting slider bar, isolated point or irregular noise were selected and then all the selected points were removed by click on Delete Data icon on toolbar. Figure 35 shows the selected slope area after data cleaning. After done with some editing, the datasets were exported in '.pts' format in order to process in CloudCompare software version 2.9.beta.

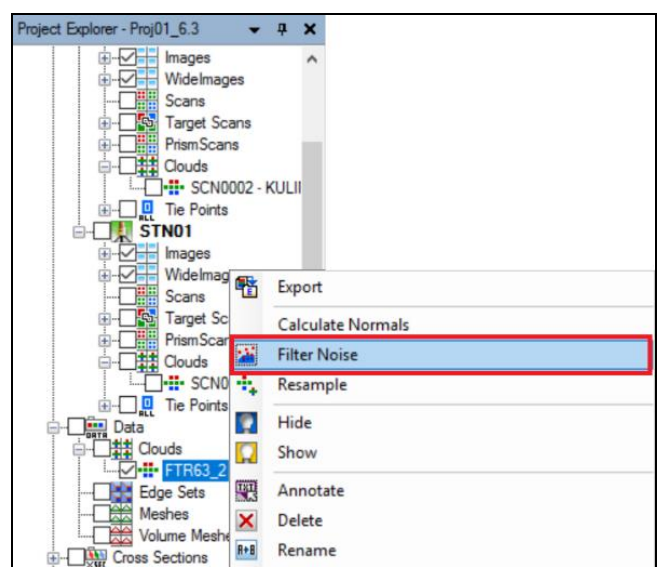

Figure 34. Filter Noise on the shortcut menu

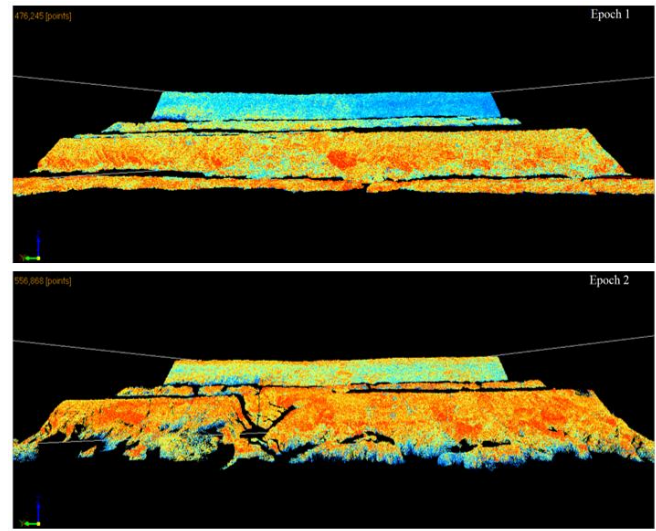

Figure 35 . The selected slope area after the data cleaning for epoch 1 and 2.

\subsubsection{Data Processing Using CloudCompare Software}

CloudCompare software is a 3D point cloud (and triangular mesh) editing and processing software. Originally, it has been designed to perform direct comparison between dense 3D point clouds. It is capable for either direct "point cloud to point cloud" comparison or "point cloud to surface" comparison. In this software, the first epoch of dataset was set as the 'Reference' which referred as reference cloud while the second epoch of dataset was set as 'Compared' which referred as the dataset that will be registered. These two epochs of point clouds datasets were registered together through align and fine registration.

The process of align (point pairs picking) registration (Figure 36) was performed by picking several pairs of equivalent points in both datasets in order to register them. In this research, the control points and target sheets near the slope were used for align registration. The equivalent points cloud was selected on both epochs at least 3 pairs. After done selected at least 3 or more pairs, CloudCompare software will display a registration report (Figure 37). However, the fine registration (Figure 38) was performed using Iterative Closest Point (ICP) algorithm in order to decrease the registration error (RMS) between the point clouds in both epochs. Fine 
registration (ICP) was used to automatically finely register two epochs of point clouds datasets.

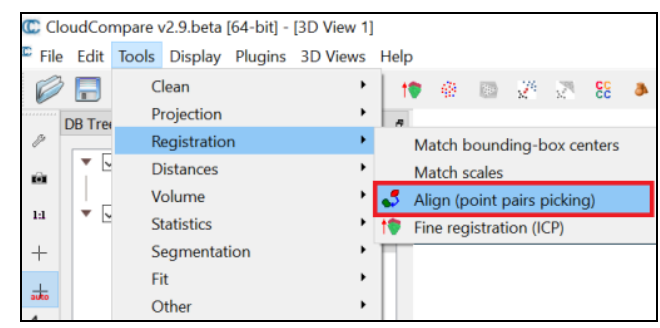

Figure 36. Align (point pairs picking) menu

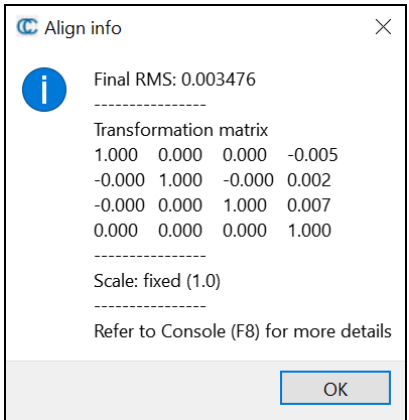

Figure 37. Registration report

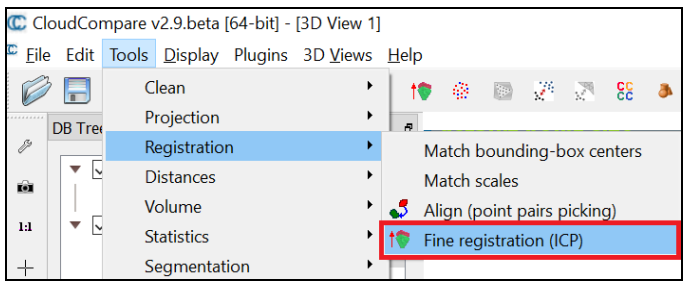

Figure 38. Fine registration (ICP) menu

After completing the process of align and fine registration, both datasets from two epochs were analysed by applying the "cloud to cloud" computation technique (Figure 39) in order to calculate the deformation of the land slope. This "cloud to cloud" computation technique calculates the nearest neighbourhood distance between two epochs using a kind of Hausdorff distance algorithm. According to GirardeauMontaut et al. (2005), the Hausdorff distance is the distance computed between the nearest points of two-point clouds. CloudCompare will compute the distances of each points relatively to the reference cloud. The Reference cloud is the cloud that will be used as reference. Figure 40 shows the example of result for cloud to cloud distances computation which the compared cloud was coloured with those approximate distances.

This section explains the methodology used to achieve the objectives of the research. There are three major phases of this methodology which are consist of fieldwork strategy, data acquisition and data processing. The fieldwork strategy in carrying out TLS fieldwork has been identified and adopted in this research after several consideration has been taken. After that, the data acquisition which were corresponding to four different types of resolution have been carried out in two epochs over the same area. Later, the data processing using

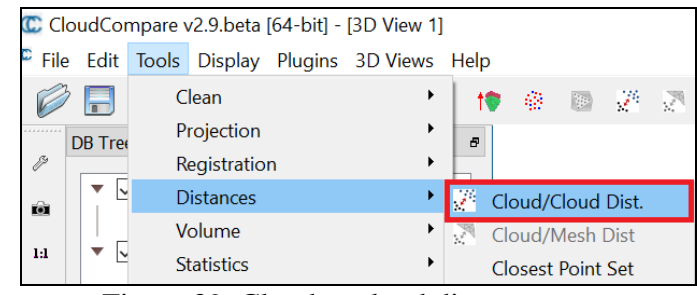

Figure 39. Cloud to cloud distance menu

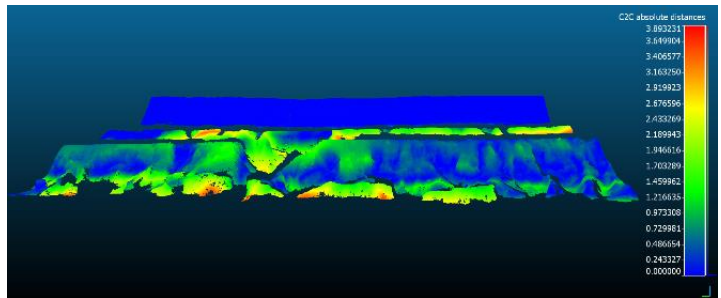

Figure 40. The example of result for cloud to cloud distances computation which was colored with those approximate distances

ScanMaster software was used to perform the data registration, georeferencing and data filtering, while the CloudCompare software was used to calculate the deformation of land slope in order to produce the displacement maps. All the results of this research were analyzed and explained in Chapter 5 in details.

\section{RESULTS, ANALYSIS AND DISCUSSION}

This chapter discussed the approaches of data analysis which conducted in this research. These include validation of TLS data and displacement map which are corresponding to four different scan resolutions. In TLS data validation, the data were analyzed through two parts which comprise of tie point constraints value evaluation and several comparison tasks of TLS point clouds dataset. Meanwhile, the displacement map was generated and analyzed using CloudCompare open source software.

\subsection{TLS Data Validation}

In this research, four dataset of TLS point cloud from each epoch were produced which are corresponding to four different scan resolutions. The reliability of these dataset was evaluated through several tasks before generating the displacement map. Thus, the first part of the analyses conducted in this stage is based on the tie point constraints value obtained during the registration and georeferencing process. Tie point constrains value indicates the errors of the pair of equivalent tie point or overlapping of point clouds that exist in registered scan. In the second part, the dataset of TLS point clouds which are corresponding to different scanning resolution were analysed and evaluated. The evaluation can be done by obtaining the coordinates value through manual selection of point clouds directly on dataset for target scans. Then, all these coordinates value were compared to automatically calculated value by software and actual coordinate's value from total station observation. 


\subsection{Evaluation of Registration and Georeferencing Accuracy Based on Tie Point Constraints}

Typically, the minimum number of three tie-points is being used in common TLS survey practice because it reduced the time spend in establishing GCP and scanning the targets. In this research, four tie-point from control points was used for registration process and all these tie-point was processed in four different datasets which are corresponding to four different scan resolutions were evaluated. The data evaluation was done on the tie-point constraints to obtain the registration and georeferencing errors during the data processing stage respectively (Table 5, Figure 41 and Figure 42). These values indicate the accuracy of the alignment within the scanning process and also determine the quality of the survey campaign that has been carried out in this research.

All the registration and georeferencing errors for both epoch was computed automatically by ScanMaster software have given the same values for each different dataset which are corresponding to four different scan resolutions. This is because the method that used for scanning the target scan using Topcon GLS-2000 laser scanner is automatically computed to determine the center of the target scan without requiring any setting for resolution. The resolution setting only required when scanning is performed on the selected specific area.

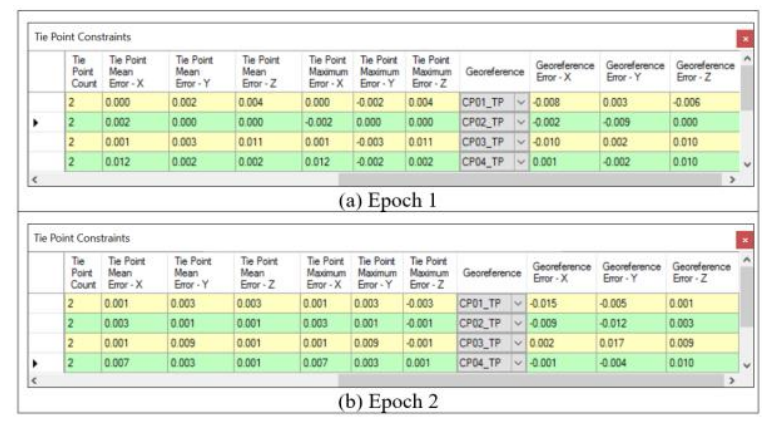

Table 5. The tie point constraint value of (a) Epoch 1 and (b) Epoch 2 for registration and georeferencing error computed by ScanMaster software

All the results as shown in Figure 41 and Figure 42 are the same as the result obtained from four different dataset which are corresponding to four different resolution. The results showed that all the tie points of each scans have been successfully aligned with minimum number of errors. By using four tie-points, the errors obtained in registration and georeferencing process are very small. Within both epochs, the maximum error detected in registration and georeferencing process are $12 \mathrm{~mm}$ and $16 \mathrm{~mm}$. These results conclude that the tie-point method implemented in this research has achieved accurate alignment within scans and the number of four tie-points used is sufficient to provide good scans alignment.

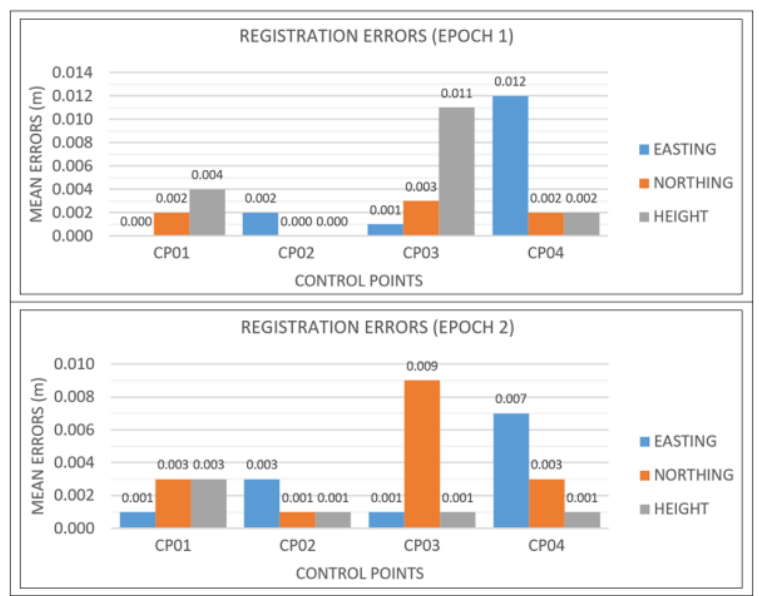

Figure 41 . The registration errors of epoch 1 and epoch 2
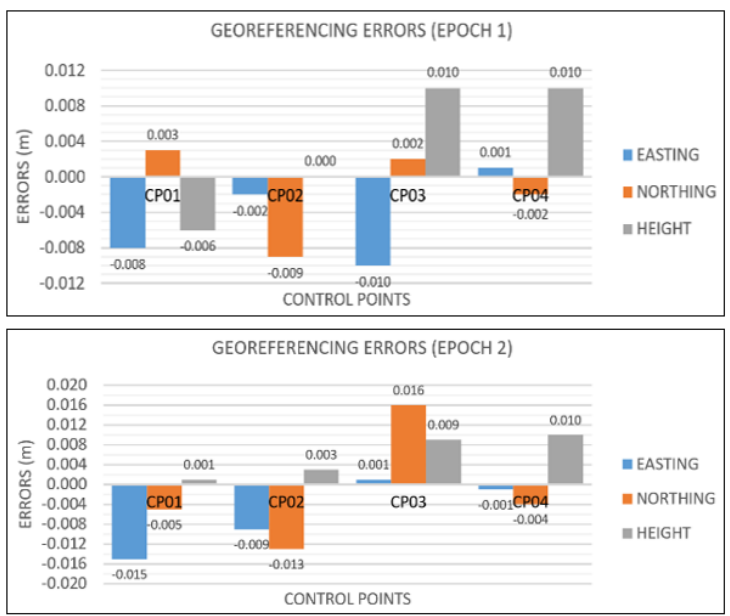

Figure 42. The georeferencing errors of epoch 1 and epoch 2

After the evaluation of registration and georeferencing process is carried out, the georeferenced point clouds were compared with all the target scans data observed by Total Station using intersection method. During the target scanning using Topcon GLS-2000 laser scanner, the resolution settings are not required in order to identify the center of the target. This is because the Topcon GLS-2000 laser scanner can determine automatically the center of the target without requiring any setting for resolution. Because of that, all the values of target scans that has been processed by ScanMaster software shows the same value for all different dataset which are corresponding to different scan resolution. Table 6 and Table 7 shows the same results were obtained for each dataset with different resolutions which are consist of low, medium, high and very high resolutions. The main objective of this analysis is to evaluate the 3D georeferenced TLS data that has been processed by ScanMaster software. All this TLS data were compared with the data observed by total station using intersection method. 


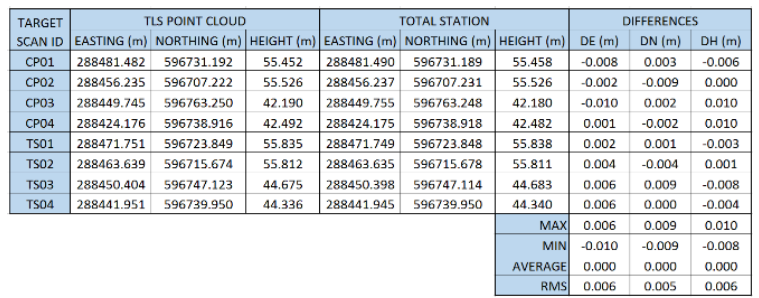

Table 6. Comparison between TLS point cloud data computed by ScanMaster software and Total Station data using intersection method (epoch 1)

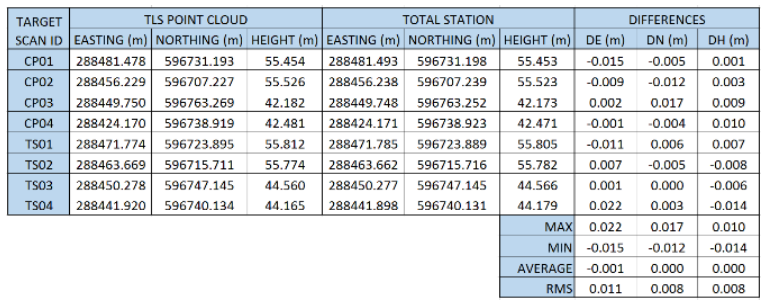

Table 7. Comparison between TLS point cloud data computed by ScanMaster software and Total Station data using intersection method (epoch 2)

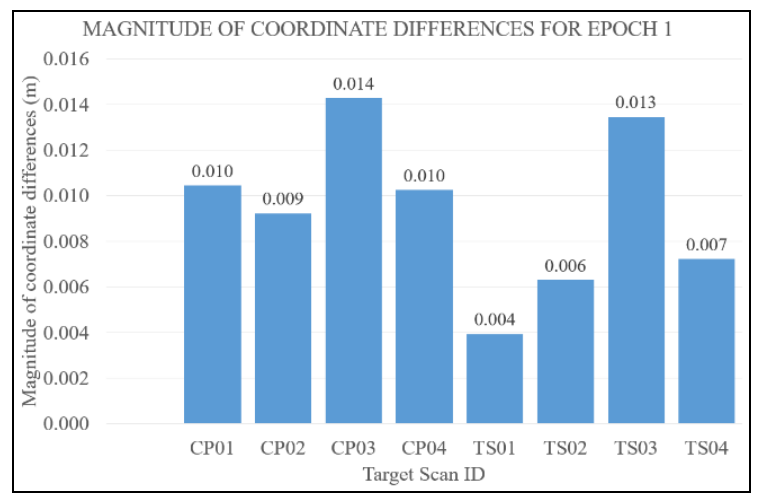

Figure 43. The magnitude of coordinate differences between TLS and Total Station data (Epoch 1)

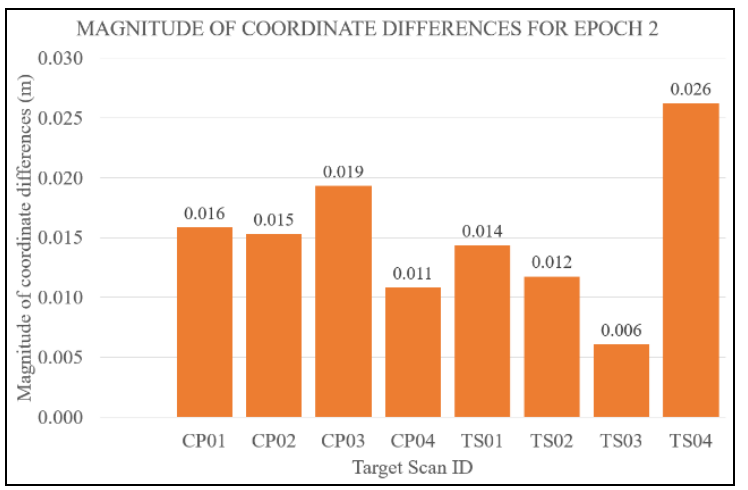

Figure 44. The magnitude of coordinate differences between TLS and Total Station data (Epoch 2)

Based on Table 6 and Table 7, it is shown that the largest coordinate differences for both epochs detected was $-10 \mathrm{~mm}$ and $22 \mathrm{~mm}$ in northing component, $9 \mathrm{~mm}$ and $-17 \mathrm{~mm}$ in easting component and $10 \mathrm{~mm}$ and $-14 \mathrm{~mm}$ in height component for epoch 1 and epoch 2 respectively. The RMS value of epoch 2 was higher than epoch 1 with value of $11 \mathrm{~mm}, 8 \mathrm{~mm}$ and $8 \mathrm{~mm}$ for northing, easting and height component respectively compared to epoch 1 with value of $6 \mathrm{~mm}, 5 \mathrm{~mm}$ and $6 \mathrm{~mm}$. The overall RMS yielded is below $11 \mathrm{~mm}$ which can be regarded as an adequate result for this research.

Meanwhile in Figure 43 and Figure 44 shows the overall result represented by magnitude component. The result shows the coordinate differences in magnitude is in range of $4 \mathrm{~mm}$ to $14 \mathrm{~mm}$ for epoch 1 and $6 \mathrm{~mm}$ to $26 \mathrm{~mm}$ for epoch 2 . Significantly, the target scan number TS04 was seemed highest among the others. The possible reason to this coordinate difference is target's instability for target sheet TS04 whereby this target was located inside the slope area which is considered unstable area. But generally, the difference magnitude for the entire points was quite good which is below $20 \mathrm{~mm}$.

\subsection{Evaluation of Four Different Types of TLS Point Cloud Data Developed from Four Different Types of Scanning Resolutions}

This section describes the task to evaluate four different types of TLS point cloud data which were developed from four different types of scanning resolution using ScanMaster software. Four different types of resolution are consisting of very high $(3.1 \mathrm{~mm}$ at $10 \mathrm{~m}$ distance $)$, high $(6.3 \mathrm{~mm}$ at $10 \mathrm{~m}$ distance), medium $(12.5 \mathrm{~mm}$ at $10 \mathrm{~m}$ distance) and low $(25 \mathrm{~mm}$ at $10 \mathrm{~m}$ distance) resolutions. The coordinate values of TLS point cloud for target scan were determined by selecting the point cloud manually. All this coordinate values were compared with the values were computed automatically by ScanMaster software and the values were obtained from Total Station observation using intersection method. The detail of each analysis task is described in following sections.

\subsubsection{Comparison between TLS Point Cloud Data by Picking Point Manually and Computed Automatically by ScanMaster Software}

This section describes the task to evaluate the differences between TLS point cloud data by picking point manually and computed automatically by ScanMaster software which are corresponding to different scan resolutions. Based on the Figure 45, it is shown that the differences range of Easting coordinates for both epoch was from $-0.017 \mathrm{~m}$ to $0.018 \mathrm{~m}$ in very high resolution, $-0.015 \mathrm{~m}$ to $0.029 \mathrm{~m}$ in high resolution, $0.053 \mathrm{~m}$ to 0.044 in medium resolution and $-0.061 \mathrm{~m}$ to $0.069 \mathrm{~m}$ in low resolution for epoch 1 and epoch 2 respectively. Based on the Figure 46, it is shown that the differences range of Northing coordinates for both epoch was from $-0.010 \mathrm{~m}$ to $0.016 \mathrm{~m}$ in very high resolution, $-0.024 \mathrm{~m}$ to $0.027 \mathrm{~m}$ in high resolution, $-0.022 \mathrm{~m}$ to $0.041 \mathrm{~m}$ in medium resolution and $-0.066 \mathrm{~m}$ to $0.054 \mathrm{~m}$ in low resolution for epoch 1 and epoch 2 respectively. Based on the Figure 47, it is shown that the differences range of Height coordinates for both epoch was from $-0.020 \mathrm{~m}$ to $0.021 \mathrm{~m}$ in very high resolution, $-0.023 \mathrm{~m}$ to $0.021 \mathrm{~m}$ in high resolution, $-0.047 \mathrm{~m}$ to $0.044 \mathrm{~m}$ in medium resolution and $-0.063 \mathrm{~m}$ to $0.057 \mathrm{~m}$ in low 
resolution for epoch 1 and epoch 2 respectively. Based on the Figure 48, it is shown that the highest RMS value of coordinate differences which were consist of Easting, Northing and Height component for both epoch was $0.011 \mathrm{~m}$, $0.009 \mathrm{~m}$ and $0.013 \mathrm{~m}$ in very high resolution, $0.014 \mathrm{~m}, 0.017 \mathrm{~m}$ and $0.015 \mathrm{~m}$ in high resolution, $0.027 \mathrm{~m}, 0.025 \mathrm{~m}$ and $0.031 \mathrm{~m}$ in medium resolution and $0.052 \mathrm{~m}, 0.043 \mathrm{~m}$ and $0.046 \mathrm{~m}$ in low resolution respectively.

From the result obtained in Figure 45, Figure 46 and Figure 47, it was found that, the different scanning resolution effected the differences of coordinate's value for both epoch. The accuracy of the coordinate's differences was depending on the high density of point cloud in high resolution.

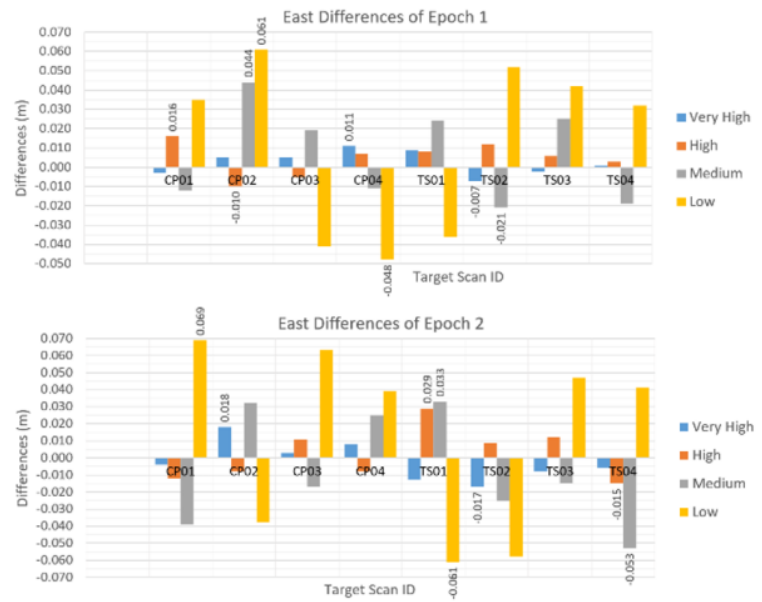

Figure 45. Easting coordinates differences between manually point picking and automatically computed by ScanMaster software (epoch 1 and 2)

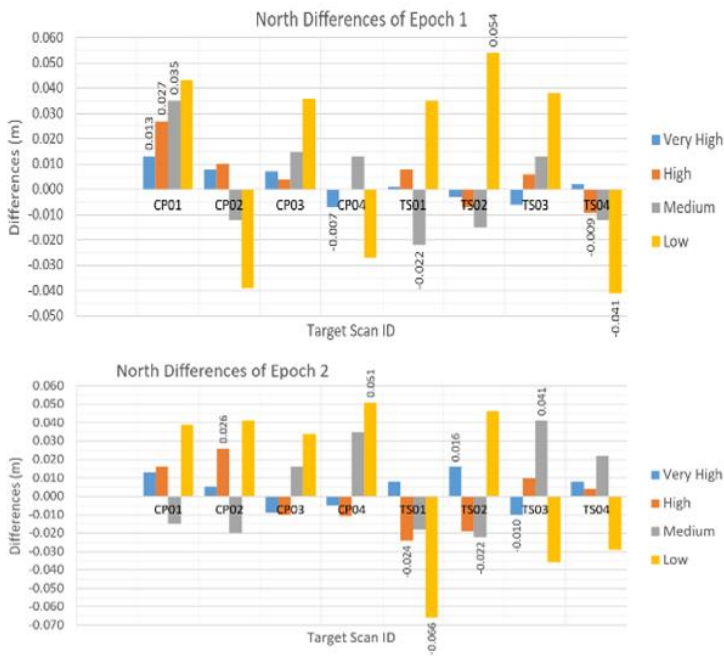

Figure 46. Northing coordinates differences between manually point picking and automatically computed by ScanMaster software (epoch 1 and 2)

In this case, the differences of coordinate's value for the very high resolution is taken as the bench mark for comparison. Hence, it can be concluded that the result of high resolution is quite close to very high resolution compared to medium and low resolutions. The Figure 48 also shows the pattern of the RMS value for coordinate differences are quite close between high and very high resolution which can provide more accurate data in slope result compared to medium and high resolutions.

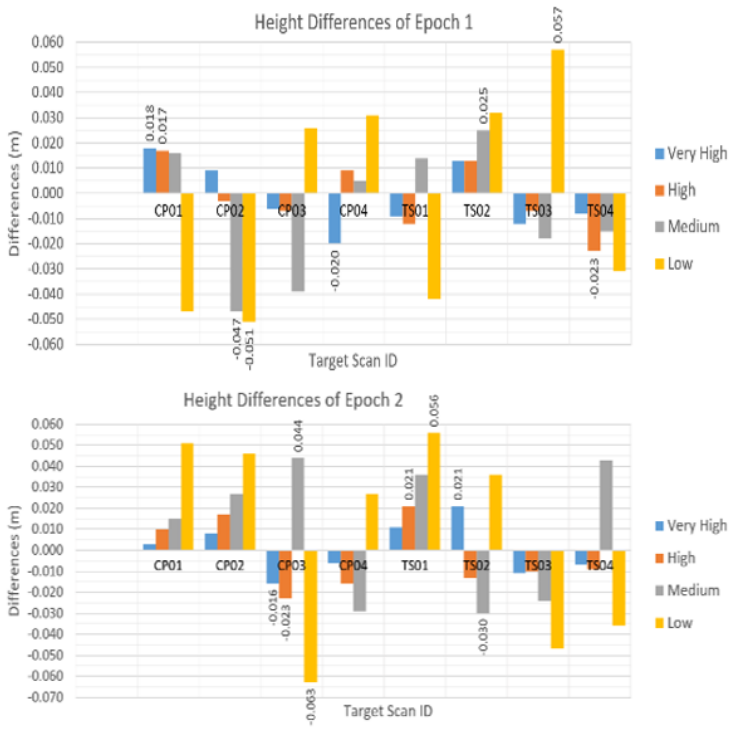

Figure 47 . Height coordinates differences between manually point picking and automatically computed by ScanMaster software (epoch 1 and 2)

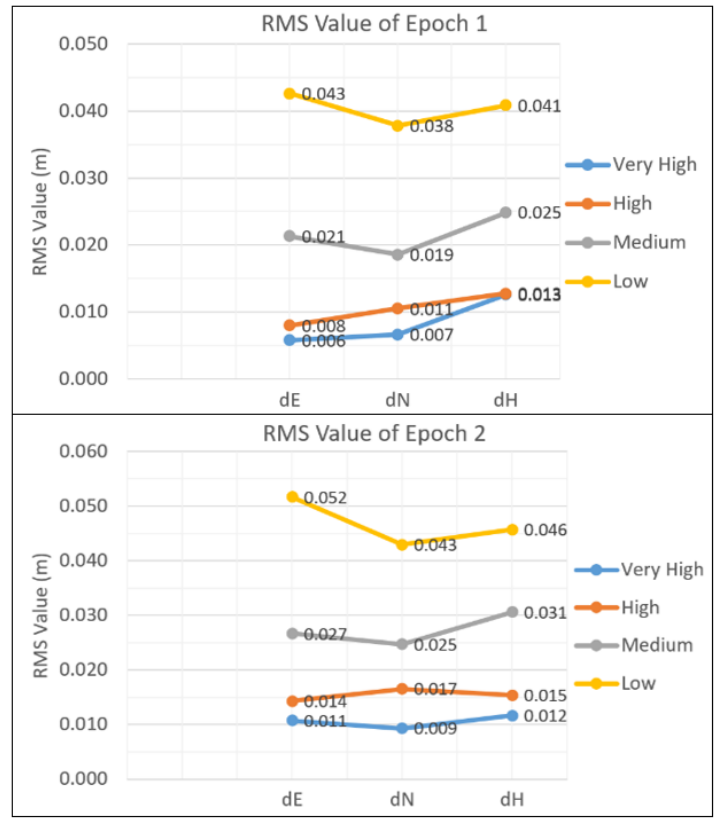

Figure 48. RMS values of coordinate differences between manually point picking and automatically computed by ScanMaster software (epoch 1 and 2) 


\subsubsection{Comparison between TLS Point Cloud Data by Picking Point Manually and Total Station Data Using Intersection Method}

This section describes the task to evaluate the differences between TLS point cloud data by picking point manually and total station data using intersection method which are corresponding to different scan resolutions. Based on the Figure 49, it is shown that the differences range of Easting coordinates for both epoch was from $-0.024 \mathrm{~m}$ to $0.016 \mathrm{~m}$ in very high resolution, $-0.027 \mathrm{~m}$ to $0.018 \mathrm{~m}$ in high resolution, $0.054 \mathrm{~m}$ to 0.042 in medium resolution and $-0.072 \mathrm{~m}$ to $0.065 \mathrm{~m}$ in low resolution for epoch 1 and epoch 2 respectively. Based on the Figure 50, it is shown that the differences range of Northing coordinates for both epoch was from $-0.010 \mathrm{~m}$ to $0.016 \mathrm{~m}$ in very high resolution, $-0.024 \mathrm{~m}$ to $0.030 \mathrm{~m}$ in high resolution, $-0.032 \mathrm{~m}$ to $0.041 \mathrm{~m}$ in medium resolution and $-0.060 \mathrm{~m}$ to $0.051 \mathrm{~m}$ in low resolution for epoch 1 and epoch 2 respectively. Based on the Figure 51, it is shown that the differences range of Height coordinates for both epoch was from $-0.021 \mathrm{~m}$ to $0.018 \mathrm{~m}$ in very high resolution, $-0.027 \mathrm{~m}$ to $0.028 \mathrm{~m}$ in high resolution, $-0.047 \mathrm{~m}$ to $0.053 \mathrm{~m}$ in medium resolution and $-0.054 \mathrm{~m}$ to $0.063 \mathrm{~m}$ in low resolution for epoch 1 and epoch 2 respectively. Based on the Figure 52, it is shown that the highest RMS value of coordinate differences which were consist of Easting, Northing and Height component for both epoch was $0.013 \mathrm{~m}$, $0.009 \mathrm{~m}$ and $0.013 \mathrm{~m}$ in very high resolution, $0.016 \mathrm{~m}, 0.014 \mathrm{~m}$ and $0.018 \mathrm{~m}$ in high resolution, $0.025 \mathrm{~m}, 0.028 \mathrm{~m}$ and $0.033 \mathrm{~m}$ in medium resolution and $0.054 \mathrm{~m}, 0.041 \mathrm{~m}$ and $0.048 \mathrm{~m}$ in low resolution respectively.

From the result obtained in Figure 49, Figure 50 and Figure 51 , it was found that, the different scanning resolution effected the differences of coordinate's value for both epoch. In this case, the differences of coordinate's value for the very high resolution is taken as the bench mark for comparison. Hence, it can be concluded that the result of high resolution is quite close to very high resolution compared to medium and low resolutions.

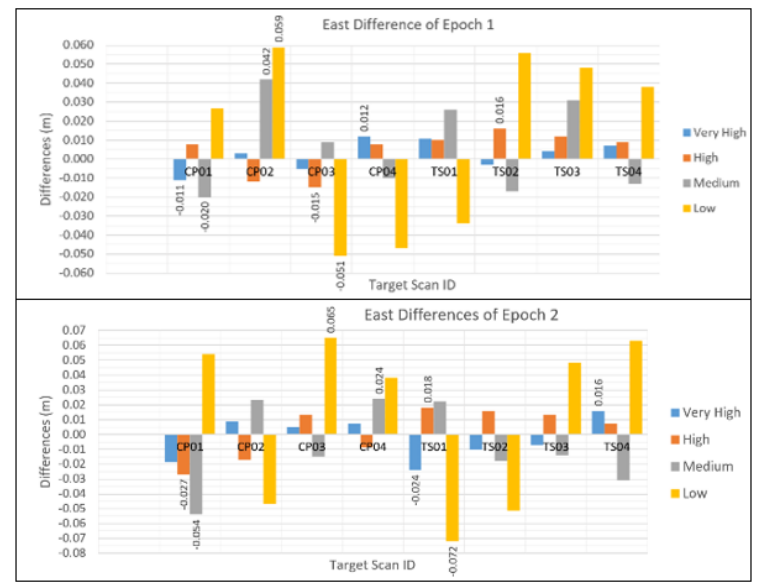

Figure 49. Easting coordinates differences between point cloud data by picking point manually and Total Station data using intersection method (epoch 1 and 2)

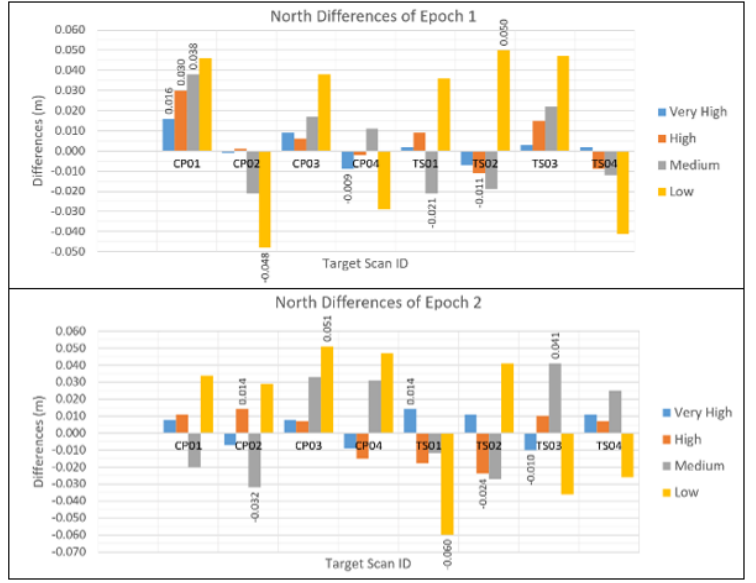

Figure 50. Northing coordinates differences between point cloud data by picking point manually and Total Station data using intersection method (epoch 1 and 2)

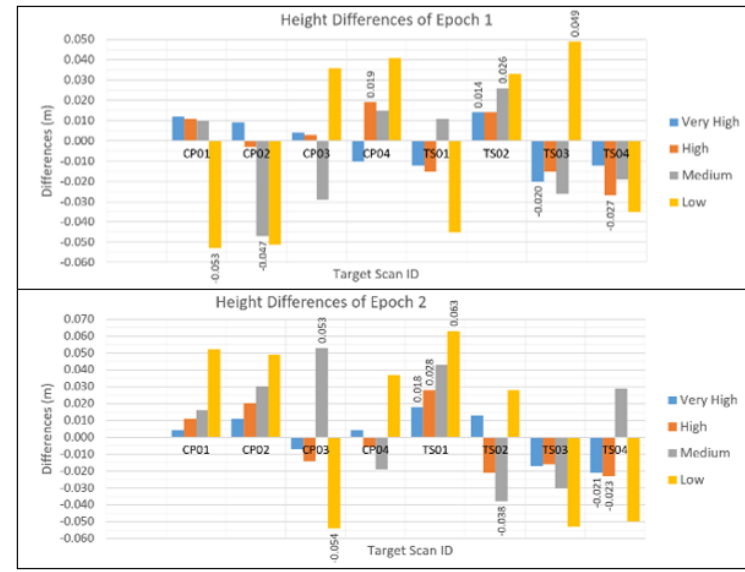

Figure 51. Height coordinates differences between point cloud data by picking point manually and Total Station data using intersection method (epoch 1 and 2)

The Figure 52 also shows the pattern of the RMS value for coordinate differences are quite close between high and very high resolution which can provide more accurate data in slope result compared to medium and high resolutions.

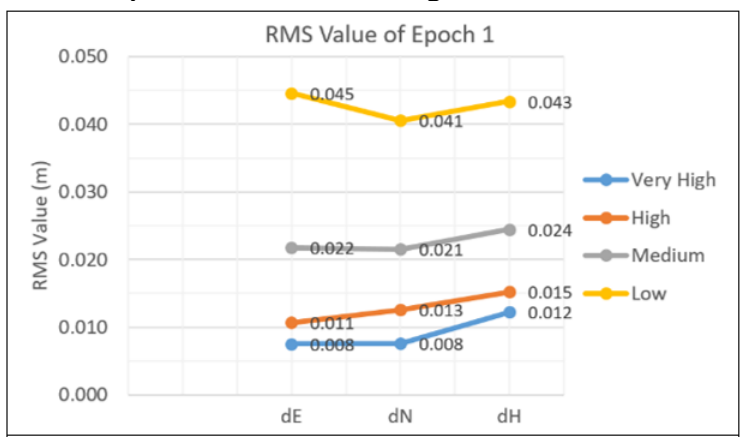




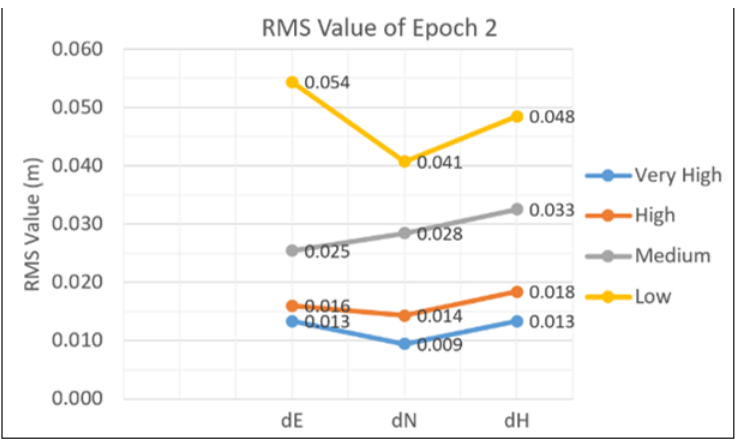

Figure 52. RMS values of coordinate differences between point cloud data by picking point manually and Total Station data using intersection method (epoch 1 and 2)

\subsubsection{The Colour-Coded Displacement Maps Using CloudCompare Software.}

This section discusses about the analysis carried out to detect the displacement of the slope area which are corresponding to different scan resolutions. There are four colour-coded displacement maps (blue, green, yellow and red) generated from four different types of scanning resolution using CloudCompare software and they are shown in Figure 53. These displacement maps were analysed by applying the "cloud to cloud" computation technique in order to calculate the deformation of the land slope. This "cloud to cloud" computation technique calculates the nearest neighbourhood distance between two epochs. The RMS values for point-pair registration error between both epochs with respect to different scan resolutions were also provided in the Figure 53.

As shown in the Figure 53, the displacement map of the slope area between both epochs have been obtained which are corresponding to different scan resolutions. It can be seen that the displacement map of high and very high resolutions can clearly detected the specific area of the landslide area compared with medium and low resolutions. The blue area indicates no movement (or stable area), while the green, yellow and red area indicates the movement of slope area. Hence, the highest of the displacement detected in all the displacement maps is around 4 meters. Besides that, from the results obtained, it can be seen that the RMS value of the point-pair registration error between both epochs is proportional to the type of different scan resolution. In this research, the RMS value of the point-pair registration error for the very high resolution is taken as the bench mark for comparison. This result indicates that the RMS value of the point-pair registration error for high resolution is quite close to very high resolution compared to medium and low resolutions. Hence, it can be concluded that both low and medium scan resolutions is not suitable for slope deformation detection.

Based on the results obtained from the entire work, it was found that the different scanning resolution affects the accuracy and displacement map of landslide deformation detection especially for low and medium resolution. Hence, it can be concluded that the high or very high resolution is needed for landslide monitoring using Topcon GLS-2000, which can provide more accurate data in slope result. The next chapter of this research focuses on the conclusion and recommendation of this research.
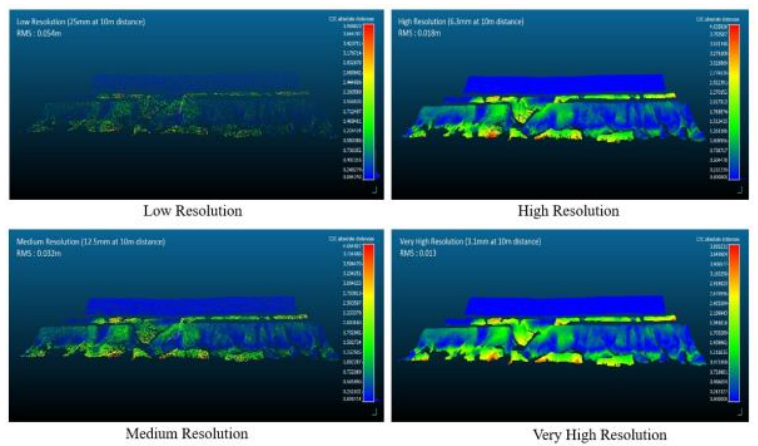

Figure 53. The generated displacement map and the RMS value for the point-pair registration error with respect to the four different scan resolutions.

\section{CONCLUSION AND RECOMMENDATION}

This research has discussed the comparison results based on the different scanning resolution using Topcon GLS-2000. Four different types of resolution are consisting of very high (3.1 $\mathrm{mm}$ at $10 \mathrm{~m}$ distance), high $(6.3 \mathrm{~mm}$ at $10 \mathrm{~m}$ distance), medium $(12.5 \mathrm{~mm}$ at $10 \mathrm{~m}$ distance) and low $(25 \mathrm{~mm}$ at $10 \mathrm{~m}$ distance) resolutions. In this research, tie-point method with indirect georeferencing was implemented due to the advantage of its accuracy to provide high quality of irregular surface model and in the same time it is easy to be conducted. Therefore, the evaluation of tie-point method in term of registration and georeferencing has been carried out. In the first evaluation, the usage of four tie-points in registration and georeferencing gave accurate alignment with $12 \mathrm{~mm}$ and $16 \mathrm{~mm}$ of maximum error. This registration and georeferencing errors gave the same result for each different dataset which are corresponding to four different scan resolutions. This is because, the registration and georeferencing errors did not affect the different scan resolution due to the method that used for scanning the target scans using Topcon GLS-2000 laser scanner is automatically computed to determine the center of the target scan without requiring any setting for resolution. The resolution setting only required when scanning is performed on the selected specific area.

The validation is then continued for reliable test whereby the different types of TLS point cloud data which were developed from four different types of scanning resolution was evaluated. To begin with, the differences between TLS point cloud data by picking point manually and computed automatically by ScanMaster software was evaluated. The finding shows the differences range of coordinates was from $0.020 \mathrm{~m}$ to $0.021 \mathrm{~m}$ in very high resolution, $-0.024 \mathrm{~m}$ to $0.029 \mathrm{~m}$ in high resolution, $-0.053 \mathrm{~m}$ to $0.044 \mathrm{~m}$ in medium resolution and $-0.066 \mathrm{~m}$ to $0.069 \mathrm{~m}$ in low resolution for epoch 1 and 2 respectively. The overall results show the different scanning resolution effected the differences of coordinate's value for both epoch especially for low resolution. The accuracy of the 
coordinate's differences was depending on the high density of point cloud in high resolution. In this case, the differences of coordinate's value for the very high resolution is taken as the bench mark for comparison. As in expectation, the result of high resolution is quite close to very high resolution compared to medium and low resolutions. Meanwhile the maximum RMS value in coordinates differences for both epoch was $0.013 \mathrm{~m}$ in very high resolution, $0.017 \mathrm{~m}$ in high resolution, $0.031 \mathrm{~m}$ in medium resolution and $0.052 \mathrm{~m}$ in low resolution respectively. The finding also shows the pattern of the RMS in coordinates difference are quite close between high and very high resolution which can provide more accurate data in slope result compared to medium and high resolutions.

In addition to validation task, the differences between TLS point cloud data by picking point manually and total station data using intersection method which are corresponding to different scan resolutions was evaluated. The finding shows the differences range of coordinates was from $-0.024 \mathrm{~m}$ to $0.018 \mathrm{~m}$ in very high resolution, $-0.027 \mathrm{~m}$ to $0.030 \mathrm{~m}$ in high resolution, $-0.054 \mathrm{~m}$ to $0.053 \mathrm{~m}$ in medium resolution and $0.072 \mathrm{~m}$ to $0.065 \mathrm{~m}$ in low resolution for epoch 1 and 2 respectively. The overall results also show the different scanning resolution effected the differences of coordinate's value for both epoch especially for low resolution. As in expectation, the result of high resolution is quite close to very high resolution compared to medium and low resolutions. Meanwhile the maximum RMS value in coordinates differences for both epoch was $0.013 \mathrm{~m}$ in very high resolution, $0.018 \mathrm{~m}$ in high resolution, $0.033 \mathrm{~m}$ in medium resolution and $0.054 \mathrm{~m}$ in low resolution respectively. The finding also shows the pattern of the RMS in coordinates difference are quite close between high and very high resolution which can provide more accurate data in slope result compared to medium and high resolutions. Hence, it can be concluded that the usage of high or higher resolution is sufficient to provide reliable end-product from TLS measurement and suitable to be implemented in the landslide research.

To complete the objective of the research, the color-coded displacement maps were analyzed to detect the displacement of the slope area which are corresponding to different scan resolutions. In the results, the displacement map of high and very high resolutions can clearly detect the specific area of the landslide area compared with medium and low resolutions. The blue area indicates no movement (or stable area), while the green, yellow and red area indicates the movement of slope area. The highest of the displacement detected in all the displacement maps is around 4 meters. The result also shows the different scanning resolution effected the RMS values of the point-pair registration error between both epochs especially for low resolution. This result indicates that the RMS value of the point-pair registration error for high resolution is quite close to very high resolution compared to medium and low resolutions. Hence, it can be concluded that both low and medium scan resolutions is not suitable for slope deformation detection due to the RMS value of the point-pair registration error will decreased when the resolution value is increased.
Based on the results obtained from the entire work, it was found that the different scanning resolution affects the accuracy and displacement map of landslide deformation detection especially for low and medium resolution. Hence, it can be concluded that the high or very high resolution is needed for landslide monitoring using Topcon GLS-2000 which can provide more accurate data in slope result. However, the very high resolution required a long observation time for collecting the point clouds data compared to high resolution. Therefore, the scanning resolution must be set according to the application needs. In addition, the displacement map of high and very high resolutions can clearly detect the specific area of the landslide area. This research also confirms that the Topcon GLS-2000 can be used for landslide deformation survey with the accuracy between $\mathrm{mm}$ and $\mathrm{cm}$ level.

The recommendations from the research conducted are as follows:

a) It is recommended to integrate both types of data acquisition methods either TLS and Unmanned Aerial Vehicle (UAV) or TLS and Airborne Laser Scanner (ALS) in order to detect the movement of landslide surface. Based on this research, the data scanning using TLS was only used to scan the slope surface and quite difficult to scan the top surface of slope due to the slope is hazardous area. The UAV or ALS method enables the data collection carried out from top surface of slope. All these data can integrate with TLS data in order to ensure that no part of the slope surface are not scanned and can provide better results in landslide monitoring survey.

b) The high resolution is also sometimes be a problem because it requires large memory space and sometimes the computer is unable to handle. However, by reducing the resolution will results to less accuracy. Therefore, the development of a suitable generalization algorithm is desired which can reduce the density of the insignificant points and at the same time maintain the accuracy.

c) Web-based GIS landslide map could be developed based on the TLS method and all data related to the research area can be combined with GIS in 3D view. Finally, these data can be used or further research.

\section{ACKNOWLEDGEMENT}

This research is part of Universiti Teknologi Malaysia (UTM) Research Grant Vot 19 H69.

\section{REFERENCES}

Abdelhafiz, A. (2009). Integrating Digital Photogrammetry and Terrestrial Laser Scanning. PhD. Thesis. Assiut University, Assiut, Egypt. 
Anne, C. W. L. (2009). Three-Dimensional Modeling of Artifact using Laser Scanner VIVID 910. Degree of Bachelor, Universiti Teknologi Malaysia, Skudai.

Bernardini, F. and Rushmeier, H. (2002). The 3D Model Acquisition Pipeline. The Eurographics Association and Blackwell Publishers.

Boehler, W. and Marbs, A. (2002). 3D Scanning Instruments. Proceedings of the CIPA WG6 International Workshop on Scanning for Cultural Heritage Recording. September 1-2. Corfu, Greece. 9-12.

Boehler, W. and Marbs, A. (2003). Investigating Laser Scanner Accuracy. Institute for Spatial Information and Surveying Technology, FH Mainz, University of Applied Sciences, Mainz, Germany.

Carr, S., Douglas, B. and Crosby, C. (2013). Terrestrial Laser Scanning (TLS) Field Camp Manual. v1.3 - 2013. UNAVCO, Boulder, CO.

Chen, W. W., Chang, C. H., Chung, M. K., Huang, P. S., Chung, W. T., Chung, Y. L. and Chen, Y. W. (2010). Landslide site reconstruction with terrestrial laser scanning. 18th International Conference on Geoinformatics

Ching, C. S. (2011). Terrestrial laser scanning for landslide (in textual) mapping. Bachelor of Engineering (Geomatic). University Technology of Malaysia, Skudai.

Girardeau-Montaut, D., Roux, M., Marc, R. and Thibault, G. (2005). Change Detection on Points Cloud Data Acquired with a Ground Laser Scanner. International Archives of Photogrammetry, Remote Sensing and Spatial Information Sciences, Commission III WG III/3, Volume 36 (part 3), W19. September 12-14. Enschede, Netherlands: ISPRS, 3035 .

Gordon, S., Lichti, D., Franke, J. and Stewart, M. (2004). Measurement of structural deformation using terrestrial laser scanners. Proceedings of FIG International Symposium on Engineering Surveys for Construction Works and Structural Engineering. 28 June-1 July. Nottingham, 16p.

Hanke, K., Grussenmeyer, P., Grimm-Pitzinger, A. and Weinold, T. (2006). First experiences with the Trimble GX scanner. International Archives of the Photogrammetry, Remote Sensing and Spatial Information Sciences, Vol. XXXVI-5, Dresden, Germany, 6 pages.

Highland, L. M. and Bobrowsky, P. (2008). The Landslide Handbook - A Guide to Understanding Landslides. Reston, Virginia: U.S. Geological Survey.

Jaboyedoff, M., Oppikofer, T., Abella'n A., Derron, M. H., Loye, A., Metzger, R. and Pedrazzini, A. (2010). Use of LIDAR in landslide investigations: a review. Natural Hazards. 61(1)(2012). 5-28. Springer.

Karsidag, G. and Alkan, R. M. (2012). Analysis of the Accuracy of Terrestrial Laser Scanning Measurements.
Proceeding of FIG Working Week 2012 on Knowing to Manage the Territory, Protect the Environment, Evaluate the Cultural Heritage. Rome, Italy.

Lerma, J. L., Navarro, S., Cabrelles, M. and Villaverde, V. (2009). Terrestrial laser scanning and close range photogrammetry for $3 D$ archaeological documentation: the Upper Palaeolithic Cave of Parpallo' as a case research. Journal of Archaeological Science, 37, 499-507. Elsevier.

Miyazaki, T., Kinoshita, K. and Takagi, M. (2008). Accurate Geometric Transformation of Laser Scanner Data for Landslide Monitoring. Infrastructure Systems Engineering, Kochi University of Technology Tosayamada-city, Kami-shi, Kochi, 782-8502, Japan.

Mohd Rosydi, A. (2013). The effect of range to the terrestrial laser scanning data, case research: slopes mapping. Bachelor of Engineering (Geomatic). University Technology of Malaysia, Skudai.

Monserrat, O. and Crosetto, M. (2008). Deformation Measurement Using Terrestrial Laser Scanning Data and Least Squares 3D Surface Matching. ISPRS Journal of Photogrammetry \& Remote Sensing 63 (2008) 142 - 154.

Petrie, G. and Toth, C. K. (2008). I. Introduction to laser ranging, profiling and scanning, II. Airbone and spaceborne laser profiles and scanners, III. Terrestrial laser scanners. In Shan, J. and Toth, C. K. (eds.) (2008). Topographic laser ranging and scanning: Principles and processing. (1-127) Boca Raton:CRC Press, Taylor \& Francis.

PWD (2009). National Slope Master Plan 2009-2023. Malaysia: Slope Engineering Branch, Public Works Department Malaysia.

Reshetyuk, Y. (2009). Self-calibration and Direct Georeferencing in Terrestrial Laser Scanning. Doctoral Thesis in Infrastructure. Royal Institute of Technology (KTH) Stockholm.

Roshan, B. and Christoph, H. (2010). African Cultural Heritage Sites and Landscapes Project. Seminar Paper. University of Wales. Position-IT Magazine, July 2010.

Rosmadi G., Ahmad Eziwan E. S., Abd Rahman A. L., Abd Rauf A. R., Zulkiflee A. L. and Abd Manan S. (2011). Evaluating the relationship between scanning resolution of laser scanner with the accuracy of the $3 D$ model constructed. 2011 IEEE International Conference on Control System, Computing and Engineering.

Schneider, D. (2006). Terrestrial Laser Scanning for Area Based Deformation Analysis of Towers and Water Dams. Proceedings of 3rd IAG/12th FIG Symp. May 22-24. Baden, Austria.10p.

Soudarissanane, S., Lindenbergh, R., Menenti, M. and Teunissen, P. (2011). Scanning geometry: Influencing factor on the quality of terrestrial laser scanning points. ISPRS 
Journal of Photogrammetry and Remote Sensing 66(4), pp. 389-399.

Sui, L., Wang, X., Zhao, D., and Qu, J. (2008). Application of $3 D$ Laser Scanner for Monitoring of Landslide Hazards. The International Archives of the Photogrammetry, Remote Sensing and Spatial Information Sciences. Vol. XXXVII. Part B1. Beijing.

Syahmi, M. Z. (2013). Three Dimensional Surface Movement Detection Using Terrestrial Laser Scanning Method, Master of Science (Geomatic Engineering). University Technology of Malaysia, Skudai.

Topcon Corporation (2014). Instruction manual laser scanner GLS-2000 series. Tokyo, Japan. from https://www.topconpositioning.com

Topcon Corporation (2015). GLS-2000 3D Laser Scanner. Tokyo, Japan. from http://www.topcon.co.jp

Tsakiri, M., Lichti, D. and Pfeifer, N. (2006). Terrestrial Laser Scanning For Deformation Monitoring. 3rd IAG / 12th FIG Symposium, Baden, May 22-24, 2006.

Varnes, D. J. 1978. Slope Movement Types and Processes. Transportation Research Board Special Report, 176.

Wan Aziz, W. A., Khairul Nizam, T. and Anuar, A. (2012). Slope gradient analysis at different resolution using terrestrial laser scanner. 2012 IEEE 8th International Colloquium on Signal Processing and its Applications.

Yong, K. P. (2012). Terrestrial laser scanning for monitoring slope instability. Bachelor of Engineering (Geomatic). University Technology of Malaysia, Skudai.

Zulkarnaini, M. A. (2016). Advances Engineering Survey: Theodolite Intersection (Allan Method) [PowerPoint slides].

Zulkepli, M. (2015). Terrestrial Laser Scanning: Data Acquisition and Data Processing [PowerPoint slides].

Revised August 2018 